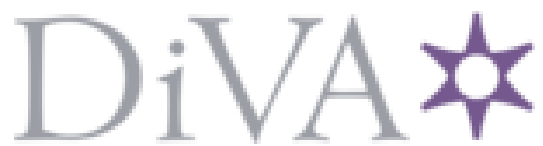

http://www.diva-portal.org

This is the published version of a paper published in Cell reports.

Citation for the original published paper (version of record):

Hernandez, S B., Dorr, T., Waldor, M K., Cava, F. (2020)

Modulation of Peptidoglycan Synthesis by Recycled Cell Wall Tetrapeptides

Cell reports, 31(4): 107578

https://doi.org/10.1016/j.celrep.2020.107578

Access to the published version may require subscription.

N.B. When citing this work, cite the original published paper.

Permanent link to this version:

http://urn.kb.se/resolve?urn=urn:nbn:se:umu:diva-170814 


\section{Cell Reports}

\section{Modulation of Peptidoglycan Synthesis by Recycled Cell Wall Tetrapeptides}

\section{Graphical Abstract}

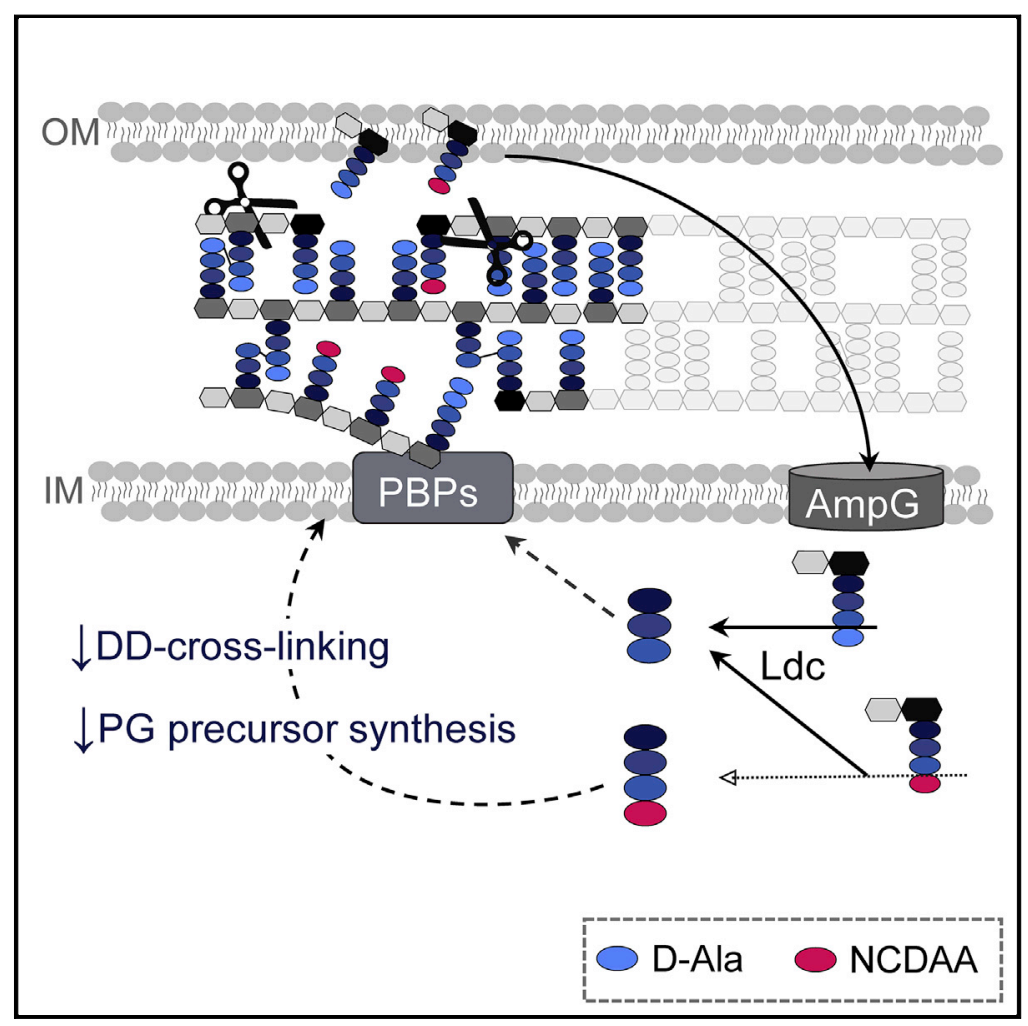

\section{Authors}

Sara B. Hernández, Tobias Dörr, Matthew K. Waldor, Felipe Cava

\section{Correspondence}

felipe.cava@umu.se

\section{In Brief}

A critical step in peptidoglycan (PG) recycling is the transformation of $P G$ tetrapeptides into tripeptides. Hernández et al. demonstrate that Vibrio cholerae accumulates tetrapeptide PG precursors to downregulate $P G$ synthesis in the stationary phase. Tetrapeptide accumulation relies on the substrate preference of L,D-carboxypeptidases for D-ala versus NCDAA-modified substrates.

\section{Highlights}

- Vibrio cholerae releases peptidoglycan (PG) fragments edited with NCDAAs

- Recycling of NCDAA-muropeptides induces the accumulation of PG-tetrapeptide precursors

- V. cholerae recycled tetrapeptides are substrates for PG synthesis

- Recycled PG-tetrapeptides control cell wall synthesis and crosslinking 


\title{
Modulation of Peptidoglycan Synthesis by Recycled Cell Wall Tetrapeptides
}

\author{
Sara B. Hernández, ${ }^{1}$ Tobias Dörr, ${ }^{2}$ Matthew K. Waldor, ${ }^{3}$ and Felipe Cava ${ }^{1,4, *}$ \\ ${ }^{1}$ The Laboratory for Molecular Infection Medicine Sweden (MIMS), Department of Molecular Biology, Umeå University, Umeå, Sweden \\ ${ }^{2}$ Department of Microbiology, Weill Institute for Cell and Molecular Biology, and Cornell Institute of Host-Microbe Interactions and Disease, \\ Cornell University, Ithaca, NY 14853, USA \\ ${ }^{3}$ Howard Hughes Medical Institute, Brigham and Women's Hospital Division of Infectious Diseases, and Department of Microbiology, Harvard \\ Medical School, Boston, MA, USA \\ ${ }^{4}$ Lead Contact \\ *Correspondence: felipe.cava@umu.se \\ https://doi.org/10.1016/j.celrep.2020.107578
}

\section{SUMMARY}

The bacterial cell wall is made of peptidoglycan (PG), a polymer that is essential for the maintenance of cell shape and survival. During growth, bacteria remodel their PG, releasing fragments that are predominantly reinternalized and recycled. Here, we show that Vibrio cholerae recycles PG fragments modified with non-canonical D-amino acids (NCDAA), which lead to the accumulation of cytosolic PG tetrapeptides. We demonstrate that the accumulation of recycled tetrapeptides has two regulatory consequences for the cell wall: reduction of D,D-cross-linkage and reduction of PG synthesis. We further demonstrate that L,D-carboxypeptidases from five different species show a preferential activity for substrates containing canonical (D-alanine) versus non-canonical (D-methionine) D-amino acids, suggesting that the accumulation of intracellular tetrapeptides in NCDAA-rich environments is widespread. Collectively, this work reveals a regulatory role of NCDAA linking PG recycling and synthesis to promote optimal cell wall assembly and composition in the stationary phase.

\section{INTRODUCTION}

The bacterial cell shape-determining peptidoglycan (PG) cell wall provides resistance to cell turgor pressure and protection from environmental threats (Cava and de Pedro, 2014; Vollmer et al., 2008a). PG is a heteropolymer made up of glycan chains consisting of $\mathrm{N}$-acetylglucosamine (GlcNAc) and $\mathrm{N}$-acetylmuramic acid (MurNAc). The GlcNAc-MurNAc polymers are cross-linked via short peptides, forming a cell-size mesh known as the murein sacculus (Vollmer et al., 2008a). The composition of these peptides normally includes a terminal D-alanine (D-Ala); however, this amino acid is replaced in some species by non-canonical D-amino acids (NCDAA) when cells enter the stationary growth phase (Alvarez et al., 2018; Cava et al., 2011; Lam et al., 2009).

During growth, the PG sacculus expands through the coordinated action of degradative and synthetic enzymes (Egan et al., 2015; Vollmer et al., 2008b). In Escherichia coli, 50\% of the murein sacculus is thought to be cleaved at each generation (van Heijenoort, 2011). Although some of the cleaved PG fragments are released into the extracellular medium (Boudreau et al., 2012; Goodell, 1985; Irazoki et al., 2019), most of them are transported back to the cytoplasm for their reutilization, a process referred to as the PG recycling pathway (Park and Uehara, 2008). Cleavage of the sacculus by endopeptidases (EPs) and lytic transglycosylases (LTs) releases monomeric 1,6-anhydro-muropeptides (Vollmer et al., 2008b) that are specifically im- ported into the cytoplasm by the AmpG permease (Cheng and Park, 2002; Jacobs et al., 1994) to serve as a substrate for the NagZ $\beta-N$-acetylglucosaminidase (Cheng et al., 2000; Vötsch and Templin, 2000) and the AmpD amidase (Höltje et al., 1994; Jacobs et al., 1995; Figure 1A). The resulting products of these two enzymes are further cleaved by the L,D-carboxypeptidase LdcA into tripeptides (Templin et al., 1999). The tripeptide is next transformed into uridine diphosphate-N-acetyl-muramyltripeptide (UDP-MurNAc-tripeptide) by the muropeptide ligase Mpl (Mengin-Lecreulx et al., 1996), thereby connecting PG de novo synthesis and recycling (Figure $1 \mathrm{~A}$ ).

Although PG recycling pathways are broadly conserved, this process of salvaging murein components is surprisingly not essential for bacterial growth (Park and Uehara, 2008), at least under standard laboratory conditions. Only the absence of the cytoplasmic LdcA is lethal when Escherichia coli enters into the stationary growth phase; Templin et al. (1999) suggested that the incorporation of atypical tetrapeptide PG precursors into the murein sacculus may result in a lethal cross-linkage defect because these muropeptides can only function as acceptors and not as donors in the cross-linking reaction. However, the incorporation of recycled tetrapeptide precursors into PG has not been demonstrated and the consequences of accumulation of these atypical precursors have not been explored.

Here, we show that during PG turnover Vibrio cholerae releases NCDAA-modified anhydro-murotetrapeptides, which 
A

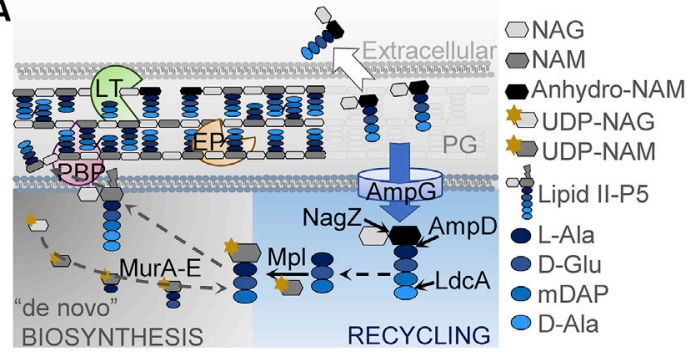

B

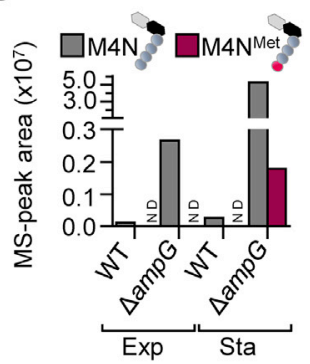

C

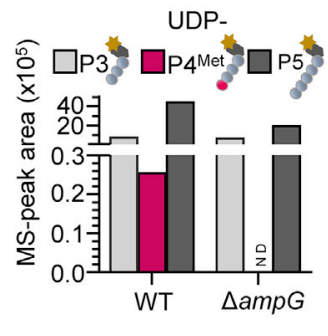

Figure 1. Recycling of Non-canonical Murotetrapeptides Leads to Production of Tetrapeptide PG Precursors (A) Schematic representation of the PG recycling pathway in E. coli (EP, endopeptidases; LT, Iytic transglycosylases; PBP, penicillin-binding proteins). (B) Extracellular anhydro-murotetrapeptides containing D-Ala (M4N) or D-Met (M4N ${ }^{M e t}$ ) at the terminal position detected by targeted MS in exponential (Exp) or stationary (Sta) cultures of indicated strains growing in LB + L-Met.

(C) UDP-muramyl-peptides (UDP-P3, UDP-P4 ${ }^{\text {Met }}$, and UDP-P5) detected by targeted MS in the cytosolic extract of stationary phase cultures of indicated strains growing in LB + L-Met. ND, not detected.

can be re-incorporated via AmpG to be re-used for PG synthesis. Recycled NCDAA-modified tetrapeptides accumulate in the stationary phase due to the preference of the recycling L,D-carboxypeptidase of $V$. cholerae (LdcV) for canonical (D-Ala) versus noncanonical (D-methionine [D-Met]) substrates, a property that is conserved in other L,D-carboxypeptidases. We further investigated the consequences of recycled tetrapeptides in the PG biosynthesis of $V$. cholerae and found that they regulate PG synthesis at two different levels: (1) reducing PG cross-linkage through their incorporation into the murein and (2) decreasing the concentration of UDP-pentapeptide precursor. Therefore, our study underscores a critical role of L,D-carboxypeptidases in the regulatory network controlling PG homeostasis and suggests that NCDAA accumulation may have evolved to circumvent L,D-carboxypeptidase activity as a means of PG architecture regulation.

\section{RESULTS}

\section{V. cholerae Recycling of Non-canonical Muropeptides} Leads to the Production of Tetrapeptide Precursors V. cholerae can synthesize different NCDAA (e.g., D-Met) through the expression of the stationary phase-dependent broad-spectrum racemase BsrV (Cava et al., 2011; Lam et al., 2009). Incorporation of NCDAA into the mature PG (primarily through periplasmic L,D-transpeptidases, Ldts) is instrumental for this bacterium to downregulate cell wall biosynthesis to adapt to growth arrest (Alvarez et al., 2018; Cava et al., 2011). Analysis of the turnover muropeptide pool of $V$. cholerae growing in the presence of L-Met revealed the presence of anhydro-murotetrapeptides with D-Met at the terminal position (M4N ${ }^{\text {Met }}$ ) (Figure 1B). M4N ${ }^{\text {Met }}$ was exclusively detected in the stationary phase and never detected in supernatants from a $\Delta b s r V$ strain (Figures $1 \mathrm{~B}$ and $\mathrm{S} 1 \mathrm{~A})$.

Recycled tetrapeptides are normally converted into tripeptides by cytosolic L,D-carboxypeptidases before they are used for the synthesis of the UDP-MurNAc-pentapeptide (UDP-P5) PG precursor (Uehara and Park, 2008; Figure 1A). However, targeted mass spectrometry (MS) analysis of $V$. cholerae stationary phase cytosolic extracts revealed the presence of
UDP-MurNAc-tetrapeptides with D-Met at the terminal position (UDP-P4 ${ }^{\mathrm{Met}}$ ) (Figures $1 \mathrm{C}$ and S1B). UDP-tetrapeptides were not detected in the $\triangle a m p G$ cytoplasm, indicating that these molecules come from recycled turnover $P G$ fragments (Figure 1C).

Identification and Characterization of the V. cholerae L,D-Carboxypeptidase Involved in Peptidoglycan

Recycling

The accumulation of UDP-MurNAc-tetrapeptide (UDP-P4) precursors in E. coli is observed after deletion of the cytosolic L,Dcarboxypeptidase LdcA (Templin et al., 1999). To investigate the biological role of recycled tetrapeptide accumulation in $V$. cholerae, we searched for the Ldc activity in this bacterium. The homolog of LdcA in V. cholerae was proposed to be VCA0337 (Templin et al., 1999). However, this protein has been reported as a microcin immunity protein (Nocek et al., 2012; Tikhonov et al., 2010), and we detected no PG hydrolytic activity for VCA0337 in vitro (Figures S2A and S2B), suggesting that Ldc in $V$. cholerae is encoded by a different gene.

Inspired by the growth phase-dependent phenotype of $\Delta / d c A$ (Templin et al., 1999), we screened an arrayed mutant library of V. cholerae (Cameron et al., 2008) to identify mutants affected in the stationary phase. We found that inactivation of the $v c 2153$ locus transformed the normal curved rod shape of the wild type into spheres only during the stationary phase (Figure 2A). The altered morphological phenotype of the $\Delta v c 2153$ mutant was accompanied by a marked reduction in PG content and a severe reduction in viability that was particularly aggravated under low osmolarity conditions (salt-free LB medium, $\mathrm{LB}_{0}$ ) (Figures 2B and 2C). VC2153 is annotated as a VanY $\mathrm{D}, \mathrm{D}$-carboxypeptidase-like protein in the NCBI database. However, using protein domain analysis (Marchler-Bauer et al., 2017), we predicted that $v c 2153$ encodes a putative L,D-carboxypeptidase similar to the Streptococcus pneumoniae LdcB. Bacterial PG carboxypeptidases are hydrolytic enzymes that remove the C-terminal amino acid from muropeptides (Vollmer et al., 2008b). While D,D-carboxypeptidases act on pentapeptides (Wright et al., 1992), L,D-carboxypeptidases use tetrapeptides as substrates (Courtin et al., 2006). VC2153 was purified to test 
A

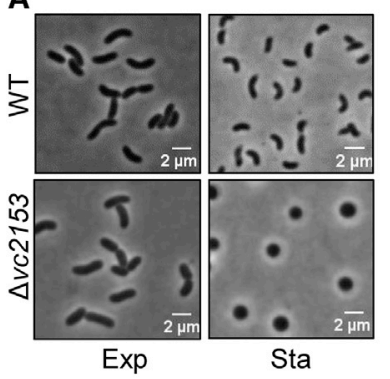

B

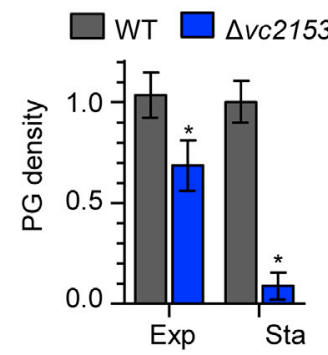

C

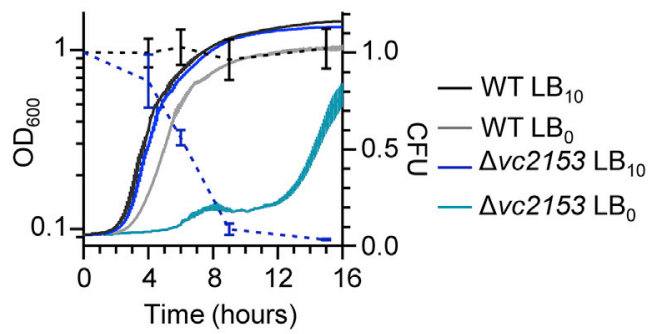

E

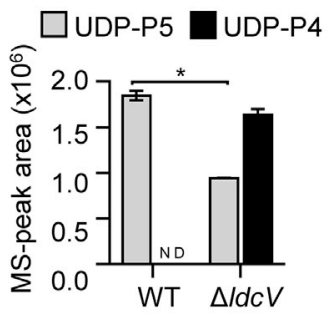

Figure 2. vc2153 Encodes an L,D-Carboxypeptidase (LdcV) That Contributes to PG Recycling in V. cholerae (A) Phase contrast microscopy of exponential (Exp) or stationary (Sta) phase LB 10 cells imaged on agarose pads.

( $B$ and C) PG density (content of PG per cell) normalized to the value of the wild type (WT) (B), growth kinetics of strains growing in LB containing 0 ( $\left(\mathrm{B} \mathrm{B}_{0}\right.$ ) or 10 ( $\left(\mathrm{B}_{10}\right)$

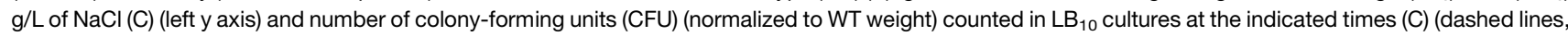
right y axis).

(D) Hydrolytic activity of purified VC2153 protein tested on isolated monomeric muropeptides (M4, murotetrapeptide; M3, murotripeptide; M5, muropentapeptide; $\mathrm{N}$, anhydro form of the muropeptide).

(E) Quantification by untargeted MS of UDP-muramyl-pentapeptide (UDP-P5) and UDP-muramyl-tetrapeptide (UDP-P4) detected in the cytosol of the indicated strains. Data represent means \pm SDs of triplicates; ND, not detected; ${ }^{*} p<0.05$, unpaired t test.

its carboxypeptidase activity using different monomeric muropeptides as potential substrates. Purified LdcA from E. coli was used as a positive control in these assays. VC2153 fully converted the disaccharide-tetrapeptide substrates (both M4 and its anhydro-derivative, M4N) to disaccharide-tripeptides (M3 and M3N), cleaving the peptide bond between mDAP and the terminal D-Ala. VC2153, like LdcA, did not act on pentapeptides (Figure 2D) or cross-linked muropeptides (e.g., D44) (Figure S2C), although in contrast to a previous report (Leguina et al., 1994), both enzymes acted on high-molecular-weight PG (Figures S2D-S2F), suggesting a potential role for L,D-carboxypeptidases in the modification of the sacculus. These observations demonstrate that VC2153 (renamed LdcV) is an L,D-carboxypeptidase in V. cholerae.

To determine whether $\mathrm{LdcV}$ is involved in $\mathrm{PG}$ recycling, we compared the pool of cytosolic UDP-activated murein precursors in the $\Delta / d c V$ mutant and the wild-type strain, using untargeted ultra-performance liquid chromatography-MS (UPLCMS). High levels of UDP-P4 (presenting D-Ala at the terminal position) were detected in the $\Delta / d c V$ mutant, but were undetectable in the wild-type strain (Figures $2 \mathrm{E}$ and S3), suggesting that LdcV is involved in the $V$. cholerae $P G$ recycling pathway. Furthermore, heterologous expression of $E$. coli IdcA under control of the $P_{\mathrm{BAD}}$ promoter complemented the growth defect of $\Delta / d c V$ (Figure S2G), indicating that LdcV is the functional homolog of the LdcA of E. coli.
L,D-Carboxypeptidases' Preference for Canonical over Non-canonical Recycled Substrates Leads to

Tetrapeptide Precursor Accumulation

We previously found that PG editing by NCDAA such as D-Met leads to the accumulation of pentapeptides in the cell wall of some bacteria, likely because these modified muropeptides are poor substrates for D,D-carboxypeptidases (Cava et al., 2011). We wondered whether NCDAA-modified muropeptides may also be suboptimal substrates during $P G$ recycling for Ldcs, explaining the accumulation of non-canonical tetrapeptide precursors (UDP-P4 ${ }^{\text {Met}}$ ) detected in $V$. cholerae wild type (Figure 1C).

To assess whether the accumulation of UDP-P4 ${ }^{\text {Met }}$ could be due to the preference of LdcV for canonical versus non-canonical tetrapeptides (D-Met modified), purified LdcV was incubated with a mixture of canonical M4N and M4 (D-Ala at the fourth position) and non-canonical M4N ${ }^{\text {Met }}$ and M4 ${ }^{\text {Met }}$ (D-Met at the fourth position) muropeptides as potential substrates. Analyses of the digestion products revealed that while $\mathrm{LdcV}$ can hydrolyze both kinds of tetrapeptides (Figure 3A), it exhibits a preference for canonical over D-Met-modified substrates (Figure 3B and S4).

Furthermore, additional L,D-carboxypeptidase in vitro assays using LdcV-like (from Aeromonas hydrophila and Proteus mirabilis) and LdcA-like (from E. coli and Salmonella enterica) enzymes exhibited a similar preference for canonical rather than NCDAAmodified murotetrapeptide substrates (Figure $3 \mathrm{C}$ ). Thus, the 
A

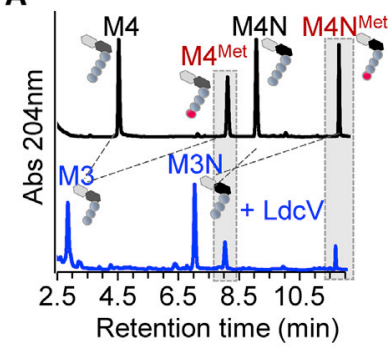

B

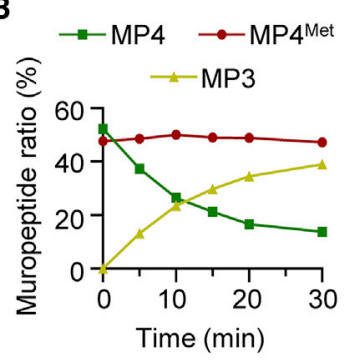

C
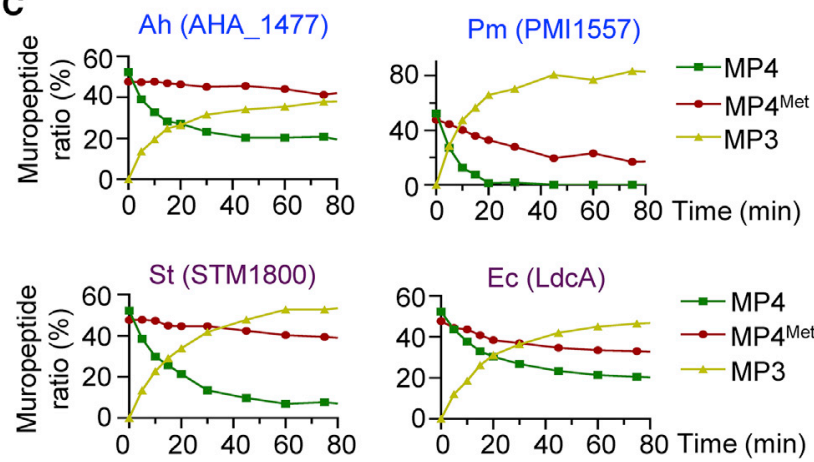

Figure 3. Peptidoglycan Recycling L,D-Carboxypeptidases Exhibit a Preference for Canonical over D-Met-Modified Murotetrapeptides (A) UPLC chromatograms showing the substrate (in black) and the resultant products (in blue) of $\mathrm{LdcV}$ in vitro assays ( $1 \mathrm{~h}$ incubation) (M4, murotetrapeptide; $\mathrm{M}^{\mathrm{Met}}$, murotetrapeptide with $\mathrm{D}-\mathrm{Met}$ at the fourth position; M4N, anhydromurotetrapeptide; $\mathrm{M} 4 \mathrm{~N}^{\mathrm{Met}}$, anhydro-murotetrapeptide with D-Met at the fourth position; $\mathrm{M} 3$, murotripeptide; $\mathrm{M} 3 \mathrm{~N}$, anhydro-murotripeptide).

(B and $C$ ) Dynamics of substrate utilization during in vitro reactions of $\mathrm{LdcV}(\mathrm{B})$ and purified L,D-carboxypeptidases from Aeromonas hydrophila (Ah), Proteus mirabilis $(\mathrm{Pm})$, Salmonella enterica serovar Typhimurium (St), and E. coli $(\mathrm{Ec})(\mathrm{C})$ $\left(M P 4=M 4+M 4 N ; M^{M P e t}=M 4^{M e t}+M 4 N^{M e t} ; M P 3=M 3+M 3 N\right)$.

production of precursor tetrapeptides during the recycling of NCDAA-edited PG seems to be a conserved mechanism.

\section{Tetrapeptide Precursors Downregulate Peptidoglycan} Synthesis

To study the regulatory role of recycled tetrapeptides in $V$. cholerae, we used the $\Delta / d c V$ mutant since this strain has increased accumulation compared to the wild type. We took advantage of the survival defect of this mutant to look for genetic determinants involved in the synthesis and function of recycled PG tetrapeptides. Colonies of the $\Delta / d c V$ strain were visibly distinct from wild type and after 3 days produced suppressors, which remained stable upon re-isolation (Figures 4A and S5A). Analyses of the suppressor colonies confirmed that they alleviated $\Delta / d c V$ phenotypes in cell morphology, PG content per cell, and growth in low osmolarity medium (Figures 4B, S5B, and $\mathrm{S} 5 \mathrm{C}$ ). The suppressor mutations were identified by whole genome sequencing (Figures S5D and S5E). To perform a more comprehensive analysis of suppressors, we carried out a transposon insertion sequencing ( $T n$-seq)-based screen in $\mathrm{LB}_{0}$ (Figures 4C and S6). Collectively, more than one-third of the suppressors corresponded to genes associated with PG recycling and turnover.

Double deletion mutants were constructed to validate and extend our suppressor analysis. Combining $\Delta / d c V$ with individual deletions of $\mathrm{PG}$ recycling genes ampG (permease), $a m p D$ (amidase), or $\mathrm{mpl}$ (muropeptide ligase) yielded complete reversion of the $\Delta / d c V$ phenotypic defects, which correlated with the absence of detectable UDP-P4 (Figures 4D-4F, S7A, and S7B). Therefore, these data suggest that the recycled tetrapeptides downregulate PG synthesis through their conversion to UDP precursors by Mpl.

However, the $\Delta / d c V \Delta$ nag $Z$ mutant accumulated UDP-P4 and incompletely suppressed the growth and $P G$ defects of the $\Delta / d c V$ mutant (Figures 4D-4F, S7A, and S7B). UDP-P4 accumulation in the nagZ IdcV mutant is likely explained by the catalytic promiscuity of AmpD (Hesek et al., 2009), which can release the tetrapeptide from both the anhydro-muramyl-peptide and M4N (accumulated in the nagZ mutant background) (Figures S7C and S7D), thereby promoting Mpl-dependent formation of UDP-P4. Similarly, deletions in PG hydrolytic enzymes only partially alleviated the $\Delta / d c V$ phenotypes and did not prevent UDP-P4 accumulation (Figures 4D-4F). Therefore, these data suggest that in addition to UDP-P4 depletion, there is a second mechanism to suppress $\Delta / d c V$ lethality.

PG hydrolases are also known as autolysins because their uncontrolled activity upon the cessation of PG synthesis can lead to cell lysis (Cavallari et al., 2013; Scheurwater et al., 2008; Shockman et al., 1996). Therefore, we hypothesized that the inactivation of these enzymes could suppress the viability defect of $\Delta / d c V$ by reducing autolysis. To monitor autolysis, we compared the amount of the PG turnover product M4N in the extracellular medium between the PG hydrolase mutants in a $\triangle a m p G$ background (to block PG recycling). There were reduced amounts of extracellular M4N in all PG hydrolase mutants (Figure 4G), suggesting that the downregulation of $P G$ synthesis by recycled tetrapeptides is balanced by PG turnover activities to maintain cell wall integrity.

\section{Recycled Tetrapeptides Can Be Incorporated into} Peptidoglycan, Leading to Reduced Cross-Linkage In agreement with a previous report (Templin et al., 1999), our data suggest that recycled tetrapeptides must be transformed into PG precursors to be active. This is further supported by the fact that most Lipid II, the final precursor for PG synthesis (Typas et al., 2011), is in the tetrapeptide form in the IdcV mutant (Figures 5A and S8). These data suggest that recycled tetrapeptides can be incorporated into the PG matrix; however, this has not been yet experimentally verified.

To address this question, we designed a strategy to distinguish between the incorporation of tetrapeptides derived from the de novo biosynthetic pathway (ending with D-Ala) and those from the recycling pathway. To this end, the growth medium was supplemented with "D-Met-labeled" anhydro-murotetrapeptide (M4N ${ }^{\text {Met }}$ ), which could be recycled and detected as a D-Met tetrapeptide in the $P G$ (Figures $5 B$ and $S 9 A$ ). To ensure that the detection of $D-M e t$ in the $P G$ exclusively relied on the incorporation of recycled tetrapeptide products, these experiments were carried out in a $\Delta / d c V$ derivative (referred to as $\Delta 4$ ) that was also deficient in endogenous D-Met production (due to the absence of the BsrV racemase) and unable to incorporate 
A

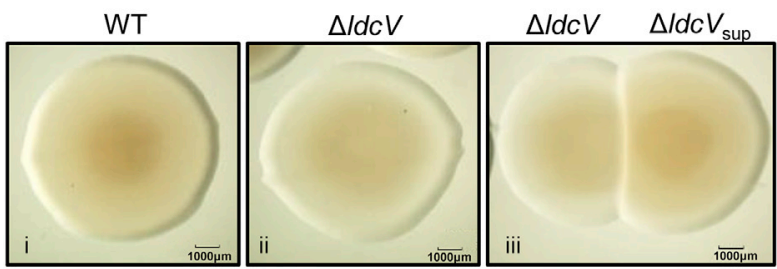

B
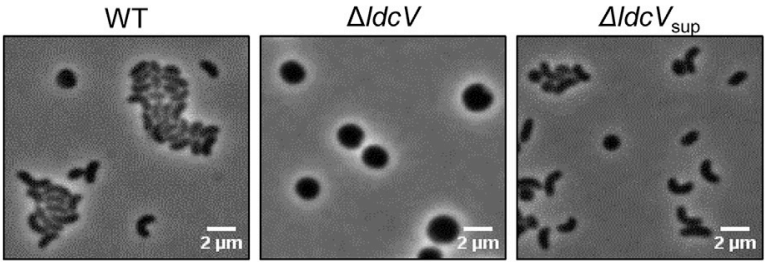

C

\begin{tabular}{|c|c|c|}
\hline Locus & Encoded product and annotated function & $\begin{array}{l}\text { Ratio reads } \\
\text { Mut/WT }\end{array}$ \\
\hline$v c 2300$ & AmpG_muropeptide transporter & 30.48 \\
\hline vc0692 & Nagz_beta-hexosaminidase & 27.15 \\
\hline vG2421 & AmpD_1,6-anhydro-N-acetylmuramyl-L-alanine amidase & $12.85 \square$ \\
\hline vc2542 & Mpl murein peptide ligase & 87.22 \\
\hline$\overline{v c 0700}$ & SIt_Soluble lytic murein transglycosylase & 13.53 \\
\hline vc2312 & MItA_membrane-bound lytic murein transglycosylase $\mathrm{A}$ & 6.82 \\
\hline vc1956 & MltB_membrane-bound lytic murein transglycosylase $B$ & 18.54 \\
\hline vc2237 & MltD_membrane-bound lytic murein transglycosylase $D$ & 4.14 \\
\hline vca0870 & PbpG_D-alanyl-D-alanine endopeptidase & 27.50 \\
\hline vca0079 & ShyA endopeptidase & 25.53 \\
\hline vc0632 & DacB D-alanyl-D-alanine carboxy/endo-peptidase & 19.31 \\
\hline
\end{tabular}

D

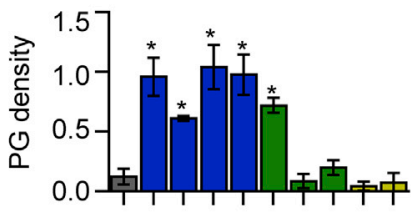

E

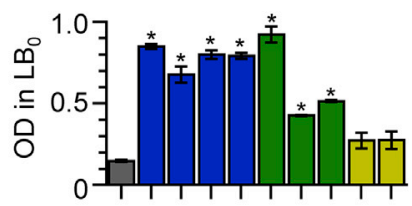

$\mathbf{F}$

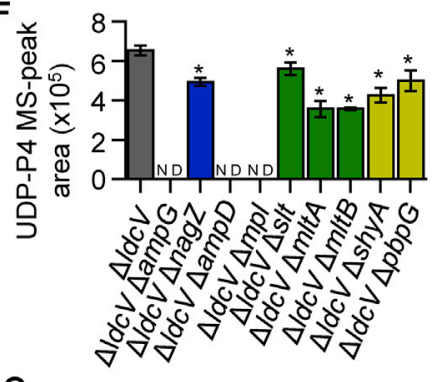

G

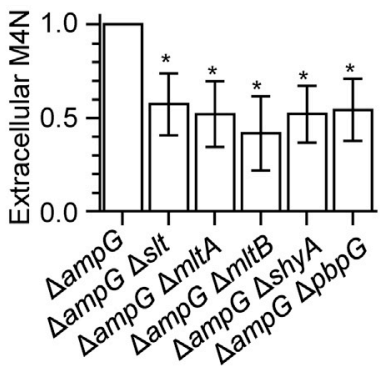

Figure 4. Phenotypes of the $\Delta / d c V$ Mutant Can Be Suppressed by the Inactivation of Genes Involved in PG Recycling and Turnover (A and B) Colony (A) and cell (B) morphology of WT, $\Delta / d c V$, and a $\Delta / d c V$ suppressor mutant ( $\Delta / d c V_{\text {sup }}$ ) growing in $\mathrm{LB}_{10}$.

(C) List of the suppressor candidate genes annotated to be involved in $P G$ homeostasis obtained from sequencing the $\Delta / d c V$-LB L $_{0} T n$ library.

(D-F) Phenotypes of $\Delta / d c V$ suppressor mutants: (D) amount of PG per cell (normalized to the WT levels) of stationary-LB 10 cultures; (E) $\mathrm{OD}_{600}$ measured after $10 \mathrm{~h}$ of incubation in $\mathrm{LB}_{0}$; and $(\mathrm{F})$ level of cytosolic UDP-P4 of the indicated mutant strains.

(G) Amount of extracellular soluble M4N (normalized to the M4N detected for the $\triangle a m p G$ single mutant) detected by untargeted MS in cultures of indicated lytic transglycosylase or endopeptidase mutants.

Data represent means \pm SDs of $2(E$ and $G)$ or $3(D$ and $F)$ biological replicates; ND, not detected; ${ }^{*} p<0.05$, unpaired $t$ test.

exogenous D-Met by L,D-transpeptidation (Cava et al., 2011) (due to the absence of LdtA and LdtB). M4 ${ }^{\text {Met }}$ was detected in the PG isolated from the $M 4 N^{M e t}$-treated $\Delta 4$ strain, but not in a control strain $(\Delta 5)$, where recycling was disabled via ampG inactivation (Figures $5 \mathrm{C}$ and $\mathrm{S} 9 \mathrm{~B}$ ). These observations demonstrate that the recycled tetrapeptides are re-used as substrates for PG synthesis.

Accumulation of tetrapeptide precursors in the $\Delta / d c V$ mutants was coupled with a marked reduction in D,D-cross-links (Figures $5 \mathrm{D}$ and $\mathrm{S10A}$ ), which is consistent with the inability of penicillinbinding proteins (PBPs) to use tetrapeptides as donor substrates in D,D-transpeptidation reactions (Vollmer et al., 2008a). Conversely, since L,D-transpeptidases use tetrapeptides instead of pentapeptides as substrates (Magnet et al., 2008; Mainardi et al., 2005; Figure 5E), we speculated that an increase in L,Dcross-links could compensate for the reduction in D,D-cross-links in the $\Delta / d c V$ mutant. Consistent with this idea, we found that an increased expression of LdtA (the main L,D-transpeptidase of $V$. cholerae) (Cava et al., 2011) improved the fitness of the $\Delta / d c V$ mutant (Figures $5 \mathrm{~F}$ and $\mathrm{S} 10 \mathrm{~B}$ ), whereas deletion of $/ d t A$ in the $\Delta / d c V$ background further attenuated its growth (Figure $5 \mathrm{G}$ ). These results demonstrate that recycled tetrapeptides control PG cross-linking homeostasis through their incorporation into the murein.

Recycled Tetrapeptides Reduce the Abundance of UDPMurNAc-Pentapeptide

Besides the reduction in PG cross-linkage, we noticed a striking inverse correlation between the accumulation of tetrapeptide (Lipid II-P4 or UDP-P4) and pentapeptide precursors (Figures $5 \mathrm{~A}$ and $6 \mathrm{~A}$ ). This result is consistent with the reduced $P G$ content of the IdcV mutant and suggests that recycled tetrapeptide precursors negatively regulate the de novo PG biosynthesis. We hypothesized that the reduction of UDP-P5 may be the result of the 
A

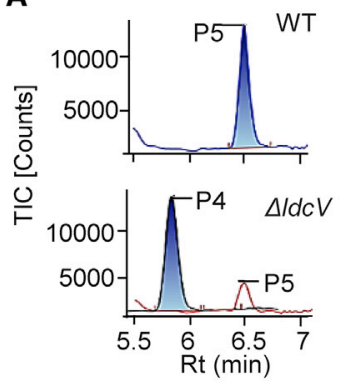

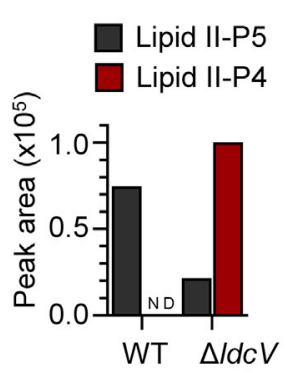
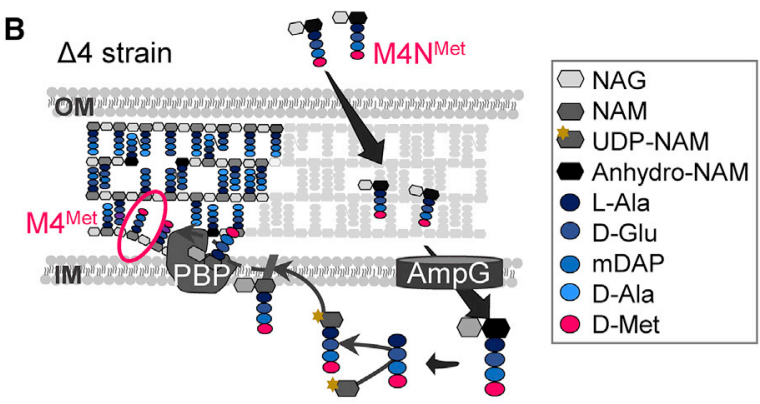

C
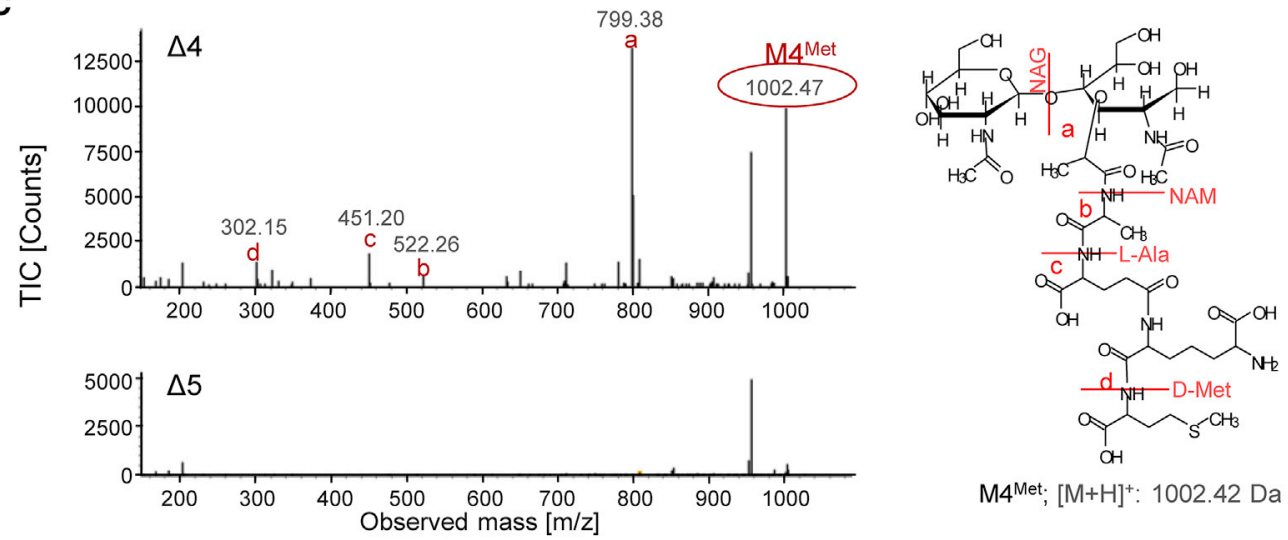

M4Met; $[\mathrm{M}+\mathrm{H}]^{+}:$1002.42 Da

D

WT background $\bullet$ IdcV background

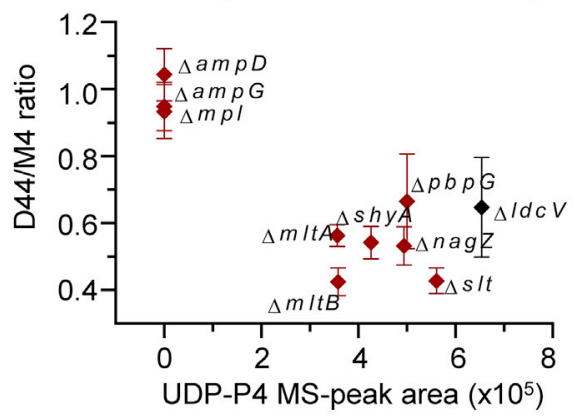

E

$\mathbf{F}$

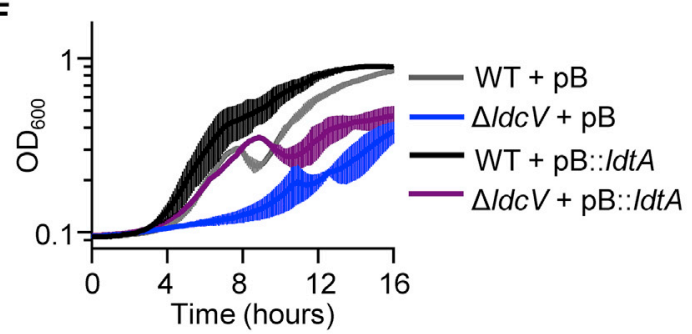

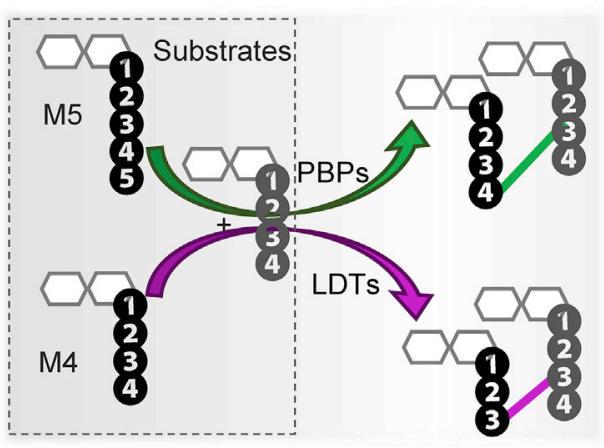

G

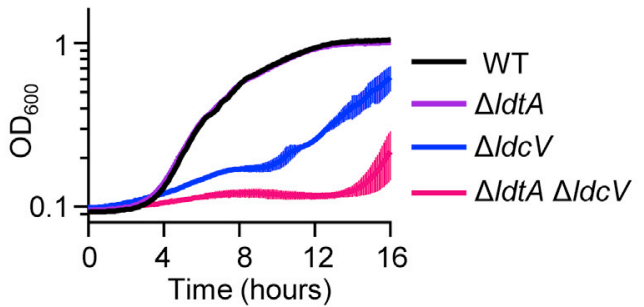

Figure 5. Incorporation of Recycled UDP-P4 into the Cell Wall Leads to a Reduction in D,D-Cross-linking (A) Detection and quantification of Lipid-II P4 and P5 delipidated forms isolated from WT and $\Delta / d c V$ cultures. ND, not detected.

(B) Schematic representation of the protocol used to demonstrate the incorporation of recycled M4N ${ }^{\text {Met }}$ into peptidoglycan. IM, inner membraned; OM, outer membrane.

(C) Exogenous M4N Met was added to cultures of $\Delta / d t A \Delta / d t B \Delta b s r V \Delta / d c V(\Delta 4)$ and $\Delta 4 \Delta a m p G(\Delta 5)$. The tandem MS (MS/MS) profile obtained by targeted MS of $\mathrm{PG}$ derived from $\Delta 4$ but not $\Delta 5$ revealed the presence of $\mathrm{M} 4^{\mathrm{Met}}$. 
A

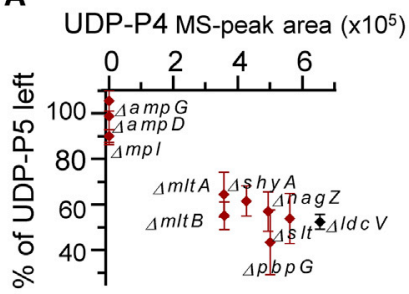

Background: $\bullet W T \bullet \triangle I d c V$
C

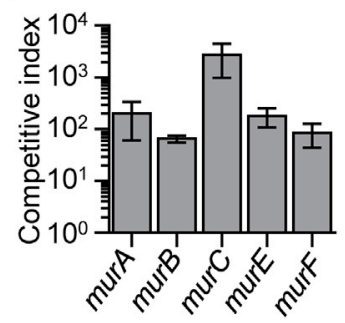

B

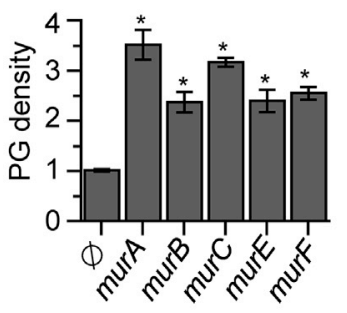

pathways for available UDP-muramyl accounts for the reduction in the amount of the UDP-P5 in the $\Delta / d c V$ mutant.

Also consistent with the hypothesis that UDP-P4 negatively regulates pentapeptide levels, a $\mathrm{Tn}$-seq-based screen for $\mathrm{IdcV}$ genetic interactions revealed a synthetic lethal/sick phenotype with the genes encoding the dominant high-molecular-weight PBP1A $(m r c A)$ and its regulatory partner proteins LpoA (Dörr et al., 2014b) and CsiV (Dörr et al., 2014a) (vc0581 and vc1887, respectively) of $V$. cholerae (Figure 6D). Collectively, these observations buttress the idea that Ldc enzymes play a key role in maintaining a proper ratio between UDP-P5 and UDP-P4 to ensure optimal PG synthesis and composition (i.e., crosslinking).

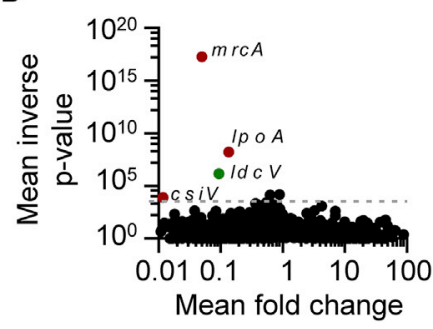

Figure 6. Tetrapeptide Precursors Negatively Regulate the De Novo PG-Biosynthetic Pathway

(A) Correlation between UDP-P5 and UDP-P4 in the indicated strains. The percentage of UDP-P5 left is calculated relative to the reference background (e.g., $\triangle a m p G \Delta / d c V$ relative to $\triangle a m p G$ ).

(B) PG density (content per cell) in $\mathrm{LB}_{10}$ of stationary cultures of the $\Delta / d c V$ strain overexpressing the indicated Mur loci from the PBAD vector. $P G$ values are relative to the strain carrying the empty vector $(\varnothing)$.

(C) Competition assay in $\mathrm{LB}_{0}$ of the $\Delta / d c V$ mutant strains overexpressing the indicated Mur loci versus the $\Delta / d c V$ mutant carrying the pBAD-empty vector. (D) Volcano plot showing the ratio of read counts mapped to individual genes in the $\Delta / d c V$ mutant $\mathrm{LB}_{10}$ transposon library compared with the control WT LB ${ }_{10}$ library. Genes represented in red and green are significantly underrepresented.

Data represent means \pm SDs of $2(B)$ or $3(A$ and $C)$ biological replicates; ${ }^{*} p<$ 0.05 , unpaired $t$ test.

UDP-muramyl substrate (the shared intermediate, i.e., UDPMurNAc named as NAM in Figure $1 A$ ) being diverted from the de novo PG synthesis pathway to UDP-P4 synthesis. Therefore, increasing the flow of the de novo pathway (i.e., the levels of UDP-P5) (Lovering et al., 2012) should alleviate the PG defect of $\Delta / d c V$. Overexpression of any of the MurA-F enzymes increased the PG density of the IdcV mutant (2-4 times) and greatly improved its fitness $(>1,000$ times in the case of MurC overexpression) in competition experiments (Figures 6B, 6C, and S10C). Interestingly, among the Mur enzymes, expression of MurC had the most potent effect on improving IdcV mutant fitness. MurC mediates the formation of UDPmuramyl-L-Ala, a substrate committed to UDP-P5, regardless of the presence of tetrapeptides. Therefore, our data suggest that competition between the de novo synthesis and recycling

\section{DISCUSSION}

The general biological importance of the PG recycling pathway is not well understood, since its inactivation has little impact on bacterial growth under laboratory conditions (Irazoki et al., 2019; Uehara and Park, 2008). Here, our investigations of the $V$. cholerae recycling pathway revealed that in stationary phase this bacterium produces NCDAA-containing PG tetrapeptide precursors in addition to the pentapeptide substrates that feed $P G$ synthesis in most bacteria. Elucidating the genetic determinants and physiological consequences of this apparent anomaly has been instrumental to uncover that L,D-carboxypeptidases exert a critical role in PG homeostasis, modulating PG synthesis and composition.

By using a $V$. cholerae $l d c$ mutant, which accumulates high levels of recycled tetrapeptides, we have demonstrated that these molecules control PG homeostasis by (1) decreasing PG cross-linkage through incorporation of activated UDP-P4 into the murein (Figure 5) and (2) downregulating PG synthesis due to the reduction of the canonical UDP-P5 precursor pool (Figure 6). The low levels of tetrapeptide precursors detected in wild-type stationary phase cells (Figure 1C) suggest that accumulation of this molecule has an actual physiological role, wherein it promotes downregulation of $\mathrm{PG}$ synthesis in order to adapt the cell envelope of $V$. cholerae to non-growth conditions in the stationary phase. As different Ldcs show distinct biochemical properties (Figure $3 C$ ), we anticipate that the levels of tetrapeptide precursors may vary between species, depending on the capacity of their Ldcs to discriminate between canonical and non-canonical substrates. For example, maximum tetrapeptide accumulation will be reached by those species that, despite possessing PG recycling pathways, do not encode L,D-carboxypeptidase orthologs (Park and Uehara, 2008).

Incorporation of recycled tetrapeptides into the murein sacculus has not been demonstrated previously. Distinguishing tetrapeptides that are generated from the activities of periplasmic PBP transpeptidases/carboxypeptidases on pentapeptide substrates from those derived from flipped Lipid II tetrapeptides

(D) Quantification of D,D-cross-linkage (estimated by calculating the D44:M4 ratio and normalized to the WT value) present in the PG of $\Delta / d c V$ suppressor mutants (y axis) and amount of accumulated UDP-P4 detected for those strains ( $x$ axis).

(E) Schematic representation of muropeptide cross-linking mediated by penicillin-binding proteins (PBPS) and L,D-transpeptidases (LDTs).

(F) Growth kinetics of indicated strains grown in $\mathrm{LB}_{0}$ supplemented with $0.2 \%$ arabinose $\left(\mathrm{pB}=\mathrm{pBAD} \mathrm{D}_{18}\right.$ ). Error bars represent $\mathrm{SD}$ of 3 biological replicates.

(G) Growth kinetics of indicated strains grown in $\mathrm{LB}_{0}$. Error bars represent SD of 3 biological replicates. 


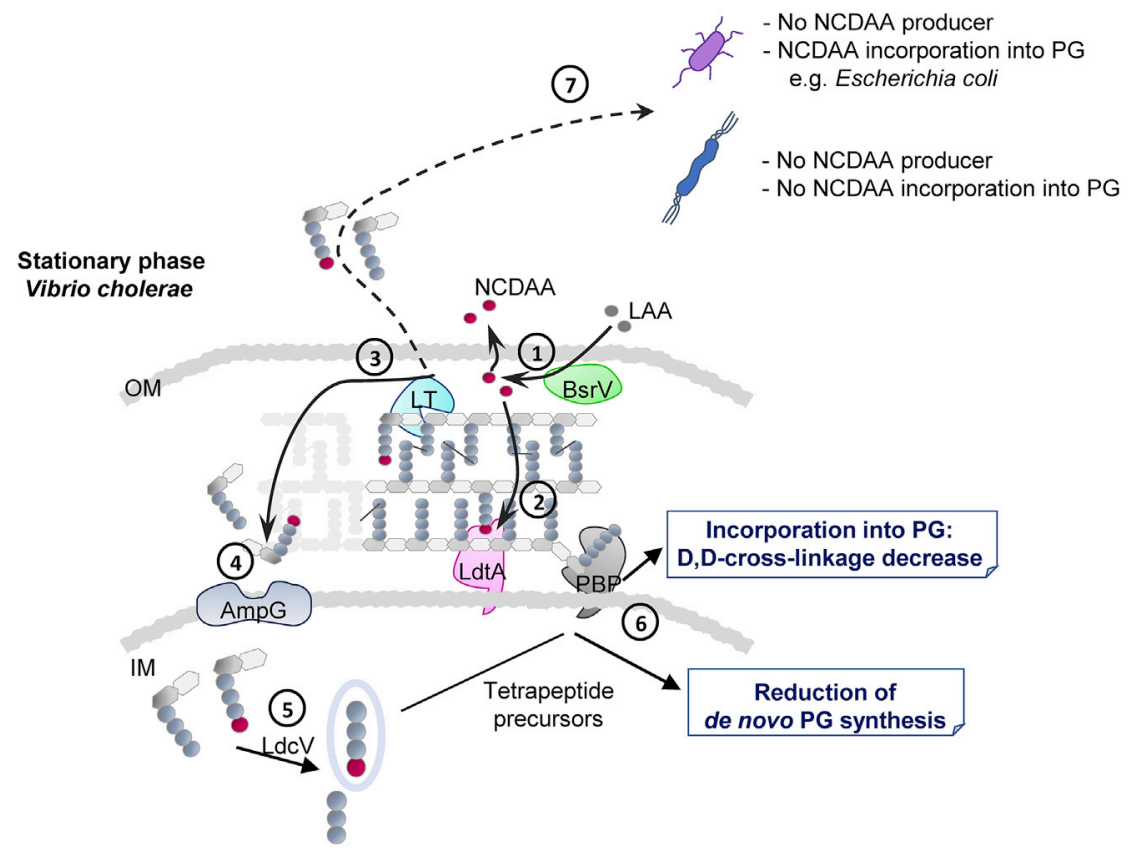

Figure 7. Schematic Representation of the Generation of PG Tetrapeptide Precursors and Their Role in V. cholerae Cell Wall Synthesis

(1) Periplasmic BsrV racemase produces NCDAA (e.g., converts L-Met into D-Met). (2) Periplasmic L,Dtranspeptidases (e.g., LdtA) incorporate D-Met into the fourth position of the peptide moiety of the muropeptides. (3) Lytic transglycosylases (LT) produce both canonical (i.e., with D-Ala) and non-canonical (e.g., with D-Met) soluble anhydro-muropeptides. (4) Anhydro-muropeptides can be recovered to the cytoplasm through the AmpG permease. (5) L,D-Carboxypeptidase (LdcV) has a preference for canonical recycled muropeptides, which leads to the accumulation of PG tetrapeptides. (6) Recycled tetrapeptides are converted into $P G$ precursors, which downregulate $P G$ synthesis and are further incorporated into the mature murein, thereby decreasing PG cross-linking. (7) Finally, released NCDAA-containing anhydro-muropeptides may also induce trans-regulatory effects in the PG synthesis or composition of bacteria inhabiting the same niche. was challenging. Nevertheless, provision of exogenous traceable D-Met anhydro-muropeptides derivatives to a $\Delta / d c V$ mutant strain incapable of synthesizing NCDAA-modified muropeptides enabled the detection of recycled tetrapeptides in the PG. The use of non-canonical anhydro-murotetrapeptides (e.g., fluorescent derivatives) could facilitate the screening of multiple species for the absence or presence of PG recycling pathways and also to assess Ldc substrate specificity in vivo. Furthermore, our findings suggest that using compounds that resemble NCDAA-modified tetrapeptides could be a means to inhibit PG biogenesis; notably, the $D$-Ser residue in the $\beta$-lactam antibiotic nocardicin $A$ is thought to specifically target Ldc enzymes (Metz et al., 1986).

Lipid-II tetrapeptide substrates can only serve as acceptors in PBP-mediated transpeptidase reactions and thus their incorporation into the sacculus results in reduced PG crosslinkage. Consistent with the incorporation of substrates unsuitable for the high-molecular-weight PBPs, we found that LdtA, an L,D-transpeptidase that uses tetrapeptides as donor substrates (Cava et al., 2011), can compensate for the growth defect of the IdcV mutant in low osmolarity (Figure 5). A number of bacterial species lack Ldc orthologs, despite apparently encoding other components of the PG recycling pathway (e.g., Acinetobacter baumanni; Park and Uehara, 2008). In organisms lacking Ldc homologs, we hypothesize that L,D-transpeptidases (Ldts) may play more prominent roles in maintaining PG homeostasis. Moreover, our data suggest that the inhibition of Ldc (e.g., with nocardicin A) in combination with inhibitors of Ldts (e.g., imipenem) (Mainardi et al., 2007) and/or PBP1a (e.g., $\beta$-lactams) may have synergistic effects and thus could represent a potent drug combination for antimicrobial therapy.

Accumulation of recycled tetrapeptides is largely prevented by cytosolic Ldc enzymes. However, NCDAA-edited tetrapepti- des (e.g., by D-Met) (Figure 1C) can accumulate since these are less preferred substrates for L,D-carboxypeptidation compared to their canonical counterparts (ending with D-Ala) (Figure 3C). The detection of UDP-tetrapeptide precursors modified with D-Met (UDP-P4 ${ }^{\mathrm{Met}}$ ) in the stationary phase wild-type cultures of $V$. cholerae (Figure 1) suggest that recycling NCDAA-modified muropeptides fine-tunes PG synthesis activity in this bacterium. Thus, production of NCDAA can modulate cell wall synthesis and cross-linking by at least two mechanisms - (1) through periplasmic PG editing (by Ldts) with free NCDAA (Alvarez et al., 2018; Cava et al., 2011; Lam et al., 2009) and (2) incorporation of recycled NCDAA-modified tetrapeptide precursors - that together coordinate cell wall synthesis with growth arrest (Figure 7). It is also possible that in addition to auto-regulatory roles, production of NCDAA induces regulatory changes in the murein of neighboring organisms. In principle, any bacterium that incorporates NCDAA in the fourth position of the muropeptide (e.g., via the activity of Ldts) is a potential source of NCDAA-modified anhydro-muropeptides that could modulate PG synthesis/composition in bacteria inhabiting the same niche (Figure 7).

Finally, extracellular PG fragments are known to be important signals in innate immunity, organ development, and behavior (Arentsen et al., 2017; Humann et al., 2016; Krueger and Opp, 2016; Mukherjee et al., 2019; Troll et al., 2009). Our observation that bacteria can release PG fragments modified with NCDAA suggests that it will be important to consider whether NCDAAmodified PG fragments convey distinct information in interkingdom signaling compared to fragments with canonical DAA. Moreover, as regards microbial ecology, our findings suggest that the release of extracellular non-canonical muropeptides could mediate interspecies modulation of PG synthesis in trans if such peptides become substrates for PG recycling in neighboring organisms. 


\section{STAR $\star$ METHODS}

Detailed methods are provided in the online version of this paper and include the following:

- KEY RESOURCES TABLE

- RESOURCE AVAILABILITY

O Lead Contact

○ Materials Availability

O Data and Code Availability

- EXPERIMENTAL MODEL AND SUBJECT DETAILS

$\circ$ Bacterial strains

O Growth conditions

O Construction of plasmids

- METHOD DETAILS

○ Cell and colony morphology imaging

o Peptidoglycan isolation and analysis

- Protein overexpression and purification

O In vitro protein reactions

o Whole genome sequencing

- Transposon insertion sequencing

- Analysis of soluble muropeptide pools

O Lipid II extraction and LC-MS analysis

O $M 4{ }^{M e t}$ incorporation into the $P G$ by recycling

- Competition assays

- QUANTIFICATION AND STATISTICAL ANALYSIS

\section{SUPPLEMENTAL INFORMATION}

Supplemental Information can be found online at https://doi.org/10.1016/j. celrep.2020.107578.

\section{ACKNOWLEDGMENTS}

We thank all of the members of the Cava lab for helpful discussions, especially Akhilesh K. Yadav and Akbar Espaillat for the support with the biochemistry part and Laura Alvarez for helping in the whole-genome sequencing (WGS) and Tn-seq analyses. We also thank Veerasak Srisuknimit for helpful comments on the manuscript. Research in the Cava lab is supported by The Swedish Research Council (VR), The Knut and Alice Wallenberg Foundation (KAW), The Laboratory of Molecular Infection Medicine Sweden (MIMS), and The Kempe Foundation. S.B.H. was supported by a Martin Escudero Postdoctoral fellowship. Research in the Dörr lab is supported by National Institutes of Health (NIH) grants R01Al143704 and R01GM130971. Research in the Waldor lab is supported by $\mathrm{NIH}$ grant R01Al-042347 and Howard Hughes Medical Institute (HHMI).

\section{AUTHOR CONTRIBUTIONS}

S.B.H. and F.C. designed and performed the research. F.C., S.B.H., T.D., and M.K.W. analyzed the data. F.C., S.B.H., T.D., and M.K.W. wrote the paper.

\section{DECLARATION OF INTERESTS}

The authors declare no competing interests.

Received: December 13, 2019

Revised: March 12, 2020

Accepted: April 6, 2020

Published: April 28, 2020

\section{REFERENCES}

Afgan, E., Baker, D., van den Beek, M., Blankenberg, D., Bouvier, D., Čech, M. Chilton, J., Clements, D., Coraor, N., Eberhard, C., et al. (2016). The Galaxy platform for accessible, reproducible and collaborative biomedical analyses: 2016 update. Nucleic Acids Res. 44 (W1), W3-W10.

Alvarez, L., Hernandez, S.B., de Pedro, M.A., and Cava, F. (2016). Ultra-Sensitive, High-Resolution Liquid Chromatography Methods for the HighThroughput Quantitative Analysis of Bacterial Cell Wall Chemistry and Structure. In Bacterial Cell Wall Homeostasis: Methods and Protocols, H.-J. Hong, ed. (Springer), pp. 11-27.

Alvarez, L., Aliashkevich, A., de Pedro, M.A., and Cava, F. (2018). Bacterial secretion of D-arginine controls environmental microbial biodiversity. ISME J. 12, 438-450.

Arentsen, T., Qian, Y., Gkotzis, S., Femenia, T., Wang, T., Udekwu, K., Forssberg, H., and Diaz Heijtz, R. (2017). The bacterial peptidoglycan-sensing molecule Pglyrp2 modulates brain development and behavior. Mol. Psychiatry $22,257-266$.

Boudreau, M.A., Fisher, J.F., and Mobashery, S. (2012). Messenger functions of the bacterial cell wall-derived muropeptides. Biochemistry 51, 2974-2990.

Cameron, D.E., Urbach, J.M., and Mekalanos, J.J. (2008). A defined transposon mutant library and its use in identifying motility genes in Vibrio cholerae. Proc. Natl. Acad. Sci. USA 105, 8736-8741.

Cava, F., and de Pedro, M.A. (2014). Peptidoglycan plasticity in bacteria: emerging variability of the murein sacculus and their associated biological functions. Curr. Opin. Microbiol. 18, 46-53.

Cava, F., de Pedro, M.A., Lam, H., Davis, B.M., and Waldor, M.K. (2011). Distinct pathways for modification of the bacterial cell wall by non-canonical D-amino acids. EMBO J. 30, 3442-3453.

Cavallari, J.F., Lamers, R.P., Scheurwater, E.M., Matos, A.L., and Burrows, L.L. (2013). Changes to its peptidoglycan-remodeling enzyme repertoire modulate $\beta$-lactam resistance in Pseudomonas aeruginosa. Antimicrob. Agents Chemother. 57, 3078-3084.

Chao, M.C., Pritchard, J.R., Zhang, Y.J., Rubin, E.J., Livny, J., Davis, B.M., and Waldor, M.K. (2013). High-resolution definition of the Vibrio cholerae essential gene set with hidden Markov model-based analyses of transposon-insertion sequencing data. Nucleic Acids Res. 41, 9033-9048.

Cheng, Q., and Park, J.T. (2002). Substrate specificity of the AmpG permease required for recycling of cell wall anhydro-muropeptides. J. Bacteriol. 184, 6434-6436.

Cheng, Q., Li, H., Merdek, K., and Park, J.T. (2000). Molecular characterization of the beta-N-acetylglucosaminidase of Escherichia coli and its role in cell wall recycling. J. Bacteriol. 182, 4836-4840.

Chiang, S.L., and Rubin, E.J. (2002). Construction of a mariner-based transposon for epitope-tagging and genomic targeting. Gene 296, 179-185.

Courtin, P., Miranda, G., Guillot, A., Wessner, F., Mézange, C., Domakova, E., Kulakauskas, S., and Chapot-Chartier, M.P. (2006). Peptidoglycan structure analysis of Lactococcus lactis reveals the presence of an L,D-carboxypeptidase involved in peptidoglycan maturation. J. Bacteriol. 188, 5293-5298.

Desmarais, S.M., De Pedro, M.A., Cava, F., and Huang, K.C. (2013). Peptidoglycan at its peaks: how chromatographic analyses can reveal bacterial cell wall structure and assembly. Mol. Microbiol. 89, 1-13.

Donnenberg, M.S., and Kaper, J.B. (1991). Construction of an eae deletion mutant of enteropathogenic Escherichia coli by using a positive-selection suicide vector. Infect. Immun. 59, 4310-4317.

Dörr, T., Lam, H., Alvarez, L., Cava, F., Davis, B.M., and Waldor, M.K. (2014a). A novel peptidoglycan binding protein crucial for PBP1A-mediated cell wall biogenesis in Vibrio cholerae. PLoS Genet. 10, e1004433.

Dörr, T., Möll, A., Chao, M.C., Cava, F., Lam, H., Davis, B.M., and Waldor, M.K. (2014b). Differential requirement for PBP1a and PBP1b in in vivo and in vitro fitness of Vibrio cholerae. Infect. Immun. 82, 2115-2124.

Dörr, T., Delgado, F., Umans, B.D., Gerding, M.A., Davis, B.M., and Waldor, M.K. (2016). A Transposon Screen Identifies Genetic Determinants of Vibrio 


\section{CellPress}

cholerae Resistance to High-Molecular-Weight Antibiotics. Antimicrob. Agents Chemother. 60, 4757-4763.

Egan, A.J., Biboy, J., van't Veer, I., Breukink, E., and Vollmer, W. (2015). Activities and regulation of peptidoglycan synthases. Philos. Trans. R. Soc. Lond. B Biol. Sci. 370, 20150031.

Espaillat, A., Forsmo, O., El Bairi, K., Bjork, R., Lemaitre, B., Trygg, J., Canada, F.J., de Pedro, M.A., and Cava, F. (2016). Chemometric Analysis of Bacterial Peptidoglycan Reveals Atypical Modifications That Empower the Cell Wall against Predatory Enzymes and Fly Innate Immunity. Journal of the American Chemical Society 138, 9193-9204.

Garrison, E., and Marth, G. (2012). Haplotype-based variant detection from short-read sequencing. arXiv, arXiv:1207.3907v2. https://arxiv.org/abs/1207. 3907.

Glauner, B., Höltje, J.V., and Schwarz, U. (1988). The composition of the murein of Escherichia coli. J. Biol. Chem. 263, 10088-10095.

Goodell, E.W. (1985). Recycling of murein by Escherichia coli. J. Bacteriol. 163, 305-310.

Guzman, L.M., Belin, D., Carson, M.J., and Beckwith, J. (1995). Tight regulation, modulation, and high-level expression by vectors containing the arabinose PBAD promoter. J. Bacteriol. 177, 4121-4130.

Heidelberg, J.F., Eisen, J.A., Nelson, W.C., Clayton, R.A., Gwinn, M.L., Dodson, R.J., Haft, D.H., Hickey, E.K., Peterson, J.D., Umayam, L., et al. (2000). DNA sequence of both chromosomes of the cholera pathogen Vibrio cholerae. Nature 406, 477-483.

Hesek, D., Lee, M., Zhang, W., Noll, B.C., and Mobashery, S. (2009). Total synthesis of $\mathrm{N}$-acetylglucosamine-1,6-anhydro- $\mathrm{N}$-acetylmuramylpentapeptide and evaluation of its turnover by AmpD from Escherichia coli. J. Am. Chem. Soc. 131, 5187-5193.

Höltje, J.V., Mirelman, D., Sharon, N., and Schwarz, U. (1975). Novel type of murein transglycosylase in Escherichia coli. J. Bacteriol. 124, 1067-1076.

Höltje, J.V., Kopp, U., Ursinus, A., and Wiedemann, B. (1994). The negative regulator of beta-lactamase induction $\mathrm{AmpD}$ is a $\mathrm{N}$-acetyl-anhydromuramylL-alanine amidase. FEMS Microbiol. Lett. 122, 159-164.

Humann, J., Mann, B., Gao, G., Moresco, P., Ramahi, J., Loh, L.N., Farr, A., Hu, Y., Durick-Eder, K., Fillon, S.A., et al. (2016). Bacterial Peptidoglycan Traverses the Placenta to Induce Fetal Neuroproliferation and Aberrant Postnatal Behavior. Cell Host Microbe 19, 901.

Irazoki, O., Hernandez, S.B., and Cava, F. (2019). Peptidoglycan Muropeptides: Release, Perception, and Functions as Signaling Molecules. Front. Microbiol. 10, 500 .

Jacobs, C., Huang, L.J., Bartowsky, E., Normark, S., and Park, J.T. (1994). Bacterial cell wall recycling provides cytosolic muropeptides as effectors for beta-lactamase induction. EMBO J. 13, 4684-4694.

Jacobs, C., Joris, B., Jamin, M., Klarsov, K., Van Beeumen, J., Mengin-Lecreulx, D., van Heijenoort, J., Park, J.T., Normark, S., and Frère, J.M. (1995). $A m p D$, essential for both beta-lactamase regulation and cell wall recycling, is a novel cytosolic N-acetylmuramyl-L-alanine amidase. Mol. Microbiol. 15, 553-559.

Koboldt, D.C., Zhang, Q., Larson, D.E., Shen, D., McLellan, M.D., Lin, L., Miller, C.A., Mardis, E.R., Ding, L., and Wilson, R.K. (2012). VarScan 2: somatic mutation and copy number alteration discovery in cancer by exome sequencing. Genome Res. 22, 568-576.

Krueger, J.M., and Opp, M.R. (2016). Sleep and Microbes. Int. Rev. Neurobiol. $131,207-225$.

Lam, H., Oh, D.C., Cava, F., Takacs, C.N., Clardy, J., de Pedro, M.A., and Waldor, M.K. (2009). D-amino acids govern stationary phase cell wall remodeling in bacteria. Science 325, 1552-1555.

Lee, M., Hesek, D., Llarrull, L.I., Lastochkin, E., Pi, H., Boggess, B., and Mobashery, S. (2013). Reactions of all Escherichia coli lytic transglycosylases with bacterial cell wall. J. Am. Chem. Soc. 135, 3311-3314.

Lee, M., Dhar, S., De Benedetti, S., Hesek, D., Boggess, B., Blázquez, B., Mathee, K., and Mobashery, S. (2016). Muropeptides in Pseudomonas aerugi- nosa and their Role as Elicitors of $\beta$-Lactam-Antibiotic Resistance. Angew. Chem. Int. Ed. Engl. 55, 6882-6886.

Leguina, J.I., Quintela, J.C., and de Pedro, M.A. (1994). Substrate specificity of Escherichia coli LD-carboxypeptidase on biosynthetically modified muropeptides. FEBS Lett. 339, 249-252.

Li, H., and Durbin, R. (2009). Fast and accurate short read alignment with Burrows-Wheeler transform. Bioinformatics 25, 1754-1760.

Lovering, A.L., Safadi, S.S., and Strynadka, N.C. (2012). Structural perspective of peptidoglycan biosynthesis and assembly. Annu. Rev. Biochem. 81, 451-478.

Magnet, S., Dubost, L., Marie, A., Arthur, M., and Gutmann, L. (2008). Identification of the L,D-transpeptidases for peptidoglycan cross-linking in Escherichia coli. J. Bacteriol. 190, 4782-4785.

Mainardi, J.L., Fourgeaud, M., Hugonnet, J.E., Dubost, L., Brouard, J.P., Ouazzani, J., Rice, L.B., Gutmann, L., and Arthur, M. (2005). A novel peptidoglycan cross-linking enzyme for a beta-lactam-resistant transpeptidation pathway. J. Biol. Chem. 280, 38146-38152.

Mainardi, J.L., Hugonnet, J.E., Rusconi, F., Fourgeaud, M., Dubost, L., Moumi A.N., Delfosse, V., Mayer, C., Gutmann, L., Rice, L.B., and Arthur, M. (2007) Unexpected inhibition of peptidoglycan LD-transpeptidase from Enterococcus faecium by the beta-lactam imipenem. J. Biol. Chem. 282, 3041430422 .

Marchler-Bauer, A., Bo, Y., Han, L., He, J., Lanczycki, C.J., Lu, S., Chitsaz, F. Derbyshire, M.K., Geer, R.C., Gonzales, N.R., et al. (2017). CDD/SPARCLE: functional classification of proteins via subfamily domain architectures. Nucleic Acids Res. 45 (D1), D200-D203.

Mengin-Lecreulx, D., van Heijenoort, J., and Park, J.T. (1996). Identification of the $\mathrm{mpl}$ gene encoding UDP-N-acetylmuramate: L-alanyl-gamma-D-glutamyl-meso-diaminopimelate ligase in Escherichia coli and its role in recycling of cell wall peptidoglycan. J. Bacteriol. 178, 5347-5352.

Metz, R., Henning, S., and Hammes, W.P. (1986). LD-carboxypeptidase activity in Escherichia coli. II. Isolation, purification and characterization of the enzyme from E. coli K 12. Arch. Microbiol. 144, 181-186.

Mukherjee, T., Hovingh, E.S., Foerster, E.G., Abdel-Nour, M., Philpott, D.J., and Girardin, S.E. (2019). NOD1 and NOD2 in inflammation, immunity and disease. Arch. Biochem. Biophys. 670, 69-81.

Nelson, M.D., and Fitch, D.H. (2011). Overlap extension PCR: an efficient method for transgene construction. Methods Mol. Biol. 772, 459-470.

Nocek, B., Tikhonov, A., Babnigg, G., Gu, M., Zhou, M., Makarova, K.S., Vondenhoff, G., Van Aerschot, A., Kwon, K., Anderson, W.F., et al. (2012). Structural and functional characterization of microcin $C$ resistance peptidase MccF from Bacillus anthracis. J. Mol. Biol. 420, 366-383.

Park, J.T., and Uehara, T. (2008). How bacteria consume their own exoskeletons (turnover and recycling of cell wall peptidoglycan). Microbiol. Mol. Biol. Rev. 72, 211-227.

Pritchard, J.R., Chao, M.C., Abel, S., Davis, B.M., Baranowski, C., Zhang, Y.J. Rubin, E.J., and Waldor, M.K. (2014). ARTIST: high-resolution genome-wide assessment of fitness using transposon-insertion sequencing. PLoS Genet. 10, e1004782.

Qiao, Y., Srisuknimit, V., Rubino, F., Schaefer, K., Ruiz, N., Walker, S., and Kahne, D. (2017). Lipid II overproduction allows direct assay of transpeptidase inhibition by $\beta$-lactams. Nat. Chem. Biol. 13, 793-798.

Rutherford, K., Parkhill, J., Crook, J., Horsnell, T., Rice, P., Rajandream, M.A. and Barrell, B. (2000). Artemis: sequence visualization and annotation. Bioinformatics 16, 944-945.

Scheurwater, E., Reid, C.W., and Clarke, A.J. (2008). Lytic transglycosylases: bacterial space-making autolysins. Int. J. Biochem. Cell Biol. 40, 586-591.

Shockman, G.D., Daneo-Moore, L., Kariyama, R., and Massidda, O. (1996). Bacterial walls, peptidoglycan hydrolases, autolysins, and autolysis. Microb. Drug Resist. 2, 95-98.

Templin, M.F., Ursinus, A., and Höltje, J.V. (1999). A defect in cell wall recycling triggers autolysis during the stationary growth phase of Escherichia coli. EMBO J. 18, 4108-4117. 
Thorvaldsdóttir, H., Robinson, J.T., and Mesirov, J.P. (2013). Integrative Genomics Viewer (IGV): high-performance genomics data visualization and exploration. Brief. Bioinform. 14, 178-192.

Tikhonov, A., Kazakov, T., Semenova, E., Serebryakova, M., Vondenhoff, G., Van Aerschot, A., Reader, J.S., Govorun, V.M., and Severinov, K. (2010). The mechanism of microcin $\mathrm{C}$ resistance provided by the MccF peptidase. J. Biol. Chem. 285, 37944-37952.

Troll, J.V., Adin, D.M., Wier, A.M., Paquette, N., Silverman, N., Goldman, W.E. Stadermann, F.J., Stabb, E.V., and McFall-Ngai, M.J. (2009). Peptidoglycan induces loss of a nuclear peptidoglycan recognition protein during host tissue development in a beneficial animal-bacterial symbiosis. Cell. Microbiol. 11 1114-1127.

Typas, A., Banzhaf, M., Gross, C.A., and Vollmer, W. (2011). From the regulation of peptidoglycan synthesis to bacterial growth and morphology. Nat. Rev Microbiol. 10, 123-136.
Uehara, T., and Park, J.T. (2008). Peptidoglycan Recycling. EcoSal Plus 3. https://doi.org/10.1128/ecosalplus.4.7.1.5.

van Heijenoort, J. (2011). Peptidoglycan hydrolases of Escherichia coli. Microbiol. Mol. Biol. Rev. 75, 636-663.

Vollmer, W., Blanot, D., and de Pedro, M.A. (2008a). Peptidoglycan structure and architecture. FEMS Microbiol. Rev. 32, 149-167.

Vollmer, W., Joris, B., Charlier, P., and Foster, S. (2008b). Bacterial peptidoglycan (murein) hydrolases. FEMS Microbiol. Rev. 32, 259-286.

Vötsch, W., and Templin, M.F. (2000). Characterization of a beta -N-acetylglucosaminidase of Escherichia coli and elucidation of its role in muropeptide recycling and beta -lactamase induction. J. Biol. Chem. 275, 39032-39038.

Wright, G.D., Molinas, C., Arthur, M., Courvalin, P., and Walsh, C.T. (1992). Characterization of vanY, a DD-carboxypeptidase from vancomycin-resistant Enterococcus faecium BM4147. Antimicrob. Agents Chemother. 36, 15141518. 


\section{STAR $\star$ METHODS}

\section{KEY RESOURCES TABLE}

\begin{tabular}{|c|c|c|}
\hline REAGENT or RESOURCE & SOURCE & IDENTIFIER \\
\hline \multicolumn{3}{|l|}{ Antibodies } \\
\hline Penta-His antibody & QIAGEN & Cat\#34660; RRID:AB_2619735 \\
\hline Anti-Mouse IgG & Sigma & Cat\#A9044; RRID:AB_258431 \\
\hline \multicolumn{3}{|l|}{ Bacterial and Virus Strains } \\
\hline All bacterial strains are listed in Table S1 & This study & Table S1 \\
\hline \multicolumn{3}{|l|}{ Chemicals, Peptides, and Recombinant Proteins } \\
\hline D-Methionine & Sigma & Cat\#M9375 \\
\hline L-Methionine & Merck & Cat\#105707 \\
\hline \multicolumn{3}{|l|}{ Critical Commercial Assays } \\
\hline Ni-NTA Agarose & QIAGEN & Cat\#30210 \\
\hline Bio-Rad Protein Assay Dye Reagent Concentrate & Bio-Rad & Cat\#500-0006 \\
\hline GeneJET Genomic DNA Purification Kit & Thermo Fisher Scientific & Cat\#K0721 \\
\hline Qubit ${ }^{\circledR}$ dsDNA HS Assay Kit & Thermo Fisher Scientific & Cat\#Q32851 \\
\hline Nextera XT DNA Library Preparation Kit & Illumina & Cat\#FC-131-1024 \\
\hline MiSeq Reagent Kit v2 (50-cycles) & Illumina & Cat\#MS-102-2001 \\
\hline \multicolumn{3}{|l|}{ Deposited Data } \\
\hline \multirow[t]{2}{*}{ Tn-seq experiments_Raw data } & This study & https://www.ncbi.nlm.nih.gov \\
\hline & & BioProject ID: PRJNA623082 \\
\hline \multirow[t]{2}{*}{ WGS suppressor mutants_Raw data } & This study & https://www.ncbi.nlm.nih.gov \\
\hline & & BioProject ID: PRJNA623074 \\
\hline \multicolumn{3}{|l|}{ Oligonucleotides } \\
\hline All oligonucleotides are detailed in Tables S2-S4 & This study & Tables S2-S4 \\
\hline \multicolumn{3}{|l|}{ Recombinant DNA } \\
\hline pET28b::vc2153-6xHis & This study & FC281 \\
\hline pET28b::/dcA-6xHis & This study & FC2025 \\
\hline pET22b::vca0337-6xHis & This study & FC2662 \\
\hline pET28b::s/t70-6xHis (E. coli) & Espaillat et al., 2016 & FC1860 \\
\hline $\mathrm{pET} 28 \mathrm{~b}:: / d t A-6 \mathrm{xHis}$ & Cava et al., 2011 & FC1168 \\
\hline pET28b::AHA1477-6xHis & This study & FC2772 \\
\hline pET28b::PMI1557-6xHis & This study & FC2773 \\
\hline pET28b::STM1800-6xHis & This study & FC2774 \\
\hline $\mathrm{pBAD}_{33}:: / d c A-6 x \mathrm{His}$ & This study & FC2753 \\
\hline $\mathrm{pBAD}_{18-\mathrm{Km}}:: / d t A-6 \times \mathrm{His}$ & This study & FC2759 \\
\hline $\mathrm{pBAD}_{18-\mathrm{Km}}:: m u r A-6 x \mathrm{His}$ & This study & FC2838 \\
\hline $\mathrm{pBAD}_{18-\mathrm{Km}}: \because$ murB-6xHis & This study & FC2839 \\
\hline $\mathrm{pBAD}_{18-\mathrm{Km}}::$ murC-6xHis & This study & FC2840 \\
\hline $\mathrm{pBAD}_{18-\mathrm{Km}}:: m u r E-6 \times \mathrm{His}$ & This study & FC2841 \\
\hline $\mathrm{pBAD}_{18-\mathrm{Km}}:: m u r F-6 x \mathrm{His}$ & This study & FC2842 \\
\hline \multicolumn{3}{|l|}{ Software and Algorithms } \\
\hline GraphPad Prism & GraphPad Software, Inc. & https://www.graphpad.com \\
\hline Zeiss Zen Blue Software & Zeiss & https://www.zeiss.com/corporate/int/home.html \\
\hline ImageJ & https://imagej.nih.gov/ij/ & https://imagej.nih.gov/ij/ \\
\hline NCBI blast & https://www.ncbi.nlm.nih.gov/BLAST/ & https://www.ncbi.nlm.nih.gov/BLAST/ \\
\hline Galaxy server tools & Afgan et al., 2016 & https://usegalaxy.org/ \\
\hline
\end{tabular}




\section{RESOURCE AVAILABILITY}

\section{Lead Contact}

Further information and requests for resources and reagents should be directed to and will be fulfilled by the Lead Contact, Felipe Cava (felipe.cava@umu.se).

Materials Availability

All bacterial and plasmids generated in this study are available on request from the Lead Contact without restriction.

Data and Code Availability

Raw sequencing data from WGS and Tn-seq experiments are available at Sequence Read Archive (https://www.ncbi.nlm.nih.gov BioProject ID PRJNA623074 and PRJNA623082 respectively). This study did not generate any new code.

\section{EXPERIMENTAL MODEL AND SUBJECT DETAILS}

\section{Bacterial strains}

Vibrio cholerae is the experimental model used in this study. All $V$. cholerae strains used in this study are derivatives of the sequenced EI Tor clinical isolate N16961 (Heidelberg et al., 2000) and are listed in Table S1. V. cholerae deletion mutant strains were constructed using standard allele exchange techniques with derivatives of the suicide plasmid pCVD442 as described previously (Donnenberg and Kaper, 1991). Primers used for constructing pCVD442-derivatives (primers P1-4) and for verifying the deletions (external primers E1-2) are shown in Table S2. Overlaping extension PCR was carried out as described (Nelson and Fitch, 2011). Since the start of $v c 2153$ overlaps with the terminal 73 nucleotides of the essential locus vc2152 (Chao et al., 2013), the $\Delta v c 2153$ mutant removes $v c 2153$ nucleotides 171 to 747 . Because strains bearing $v c 2153$ mutations accumulate suppressors, the deletion of $v c 2153$ was always the final mutation introduced in strains bearing inactivation of more than one gene.

Escherichia coli strains $\mathrm{DH} 5 \alpha$ and $\mathrm{DH} 5 \alpha$ p pir were used as hosts for constructing plasmids. The strains used as template for the amplification reaction of the genes encoding the cytoplasmic L,D-carboxypeptidase of the different bacteria were: $E$. coli $\mathrm{K}-12$ MG1655, Aeromonas hydrophila subsp. hydrophila ATCC 7966, Proteus mirabilis DSM 4479 and Salmonella enterica subsp. enterica serovar Typhimurium ATCC 14028.

\section{Growth conditions}

Unless otherwise specified, bacteria were grown at $37^{\circ} \mathrm{C}$ in LB (Luria Bertani broth) medium. Agar $1.5 \%$ (w/v) was used in solid plates. Antibiotics were used at the following concentrations (per ml): streptomycin (Sm), $200 \mu \mathrm{g}$, ampicillin (Ap), $50 \mu \mathrm{g}$, carbenicillin (Cb), $50 \mu \mathrm{g}$, chloramphenicol (Cm), $20 \mu \mathrm{g}(E$. coli) and $5 \mu \mathrm{g}(\mathrm{V}$. cholerae), and kanamycin (Kn), $50 \mu \mathrm{g}$. L- or D-methionine (L/D-Met) were used at a final concentration of $20 \mathrm{mM}$.

For growth curves, stationary cultures were normalized to an optical density at $600 \mathrm{~nm}\left(\mathrm{OD}_{600}\right)$ of 1.5 , diluted $1: 100$ and used for inoculating 96 -well plates containing $180 \mu \mathrm{L}$ of fresh medium. At least three replicates per strain and condition were carried out. Measures of the $\mathrm{OD}_{600}$ for growth curves were carried at $30^{\circ} \mathrm{C}$ with shaking using an Eon Biotek microplate spectrophotometer.

\section{Construction of plasmids}

Complementation and overexpression plasmids were constructed using the primers indicated in Table S3 by amplifying the gene of

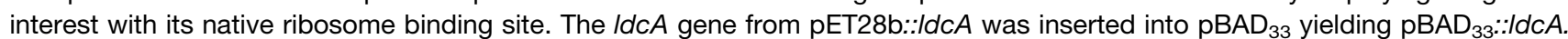
Expression of cloned loci from the $\mathrm{P}_{\mathrm{BAD}}$ promoter was induced by addition of $0.2 \%$ arabinose (Guzman et al., 1995). DNA and protein sequence alignments were carried out using the blastn and blastp programs (https://www.ncbi.nlm.nih.gov/BLAST/) respectively.

\section{METHOD DETAILS}

\section{Cell and colony morphology imaging}

Analysis of cell morphology was performed on immobilized bacteria ( $1 \%$ agarose LB pads) by phase-contrast microscopy using a Zeiss Axio Imager.Z2 microscope equipped with a $63 x$ oil immersion objective and a Hamamatsu digital camera controlled by Zeiss Zen Blue software. Pictures of colonies were taken from $\mathrm{LB}_{10}$ plates $\left(10 \mathrm{~g} \mathrm{~L}^{-1}\right.$ of NaCl) by using a Nikon SMZ1500 Zoom Stereomicroscope and a DS-Fi1 High-Definition Color Camera. Brightness and contrast levels of the images were adjusted using Image software.

\section{Peptidoglycan isolation and analysis}

Murein sacculi isolation and muropeptide analysis were performed essentially as described previously (Alvarez et al., 2016; Desmarais et al., 2013; Glauner et al., 1988). Briefly, bacterial pellets of $50 \mathrm{~mL}$ (exponential phase $\mathrm{OD}_{600} \sim 0.4$ ) or 25ml (stationary phase $\mathrm{OD}_{600} \sim 3$ ) cultures were boiled in 5\% SDS. Cell wall material was pelleted and repeatedly washed with water by ultracentrifugation. Clean sacculi were digested with muramidase $(100 \mu \mathrm{g} / \mathrm{ml})$ and soluble muropeptides were then reduced using $0.5 \mathrm{M}$ sodium borate 
$\mathrm{pH} 9.5$ and sodium borohydride at $10 \mathrm{mg} \mathrm{ml}^{-1}$ (final concentration). The $\mathrm{pH}$ of the samples was adjusted to 3.5 with phosphoric acid for liquid chromatography.

UPLC analyses were performed on a Waters-UPLC system equipped with an ACQUITY UPLC BEH C18 Column, $130 \AA$, $1.7 \mu \mathrm{m}$, $2.1 \mathrm{~mm} \times 150 \mathrm{~mm}$ (Water, USA) and detected at Abs. $204 \mathrm{~nm}$. Muropeptides were separated primarily using a linear gradient

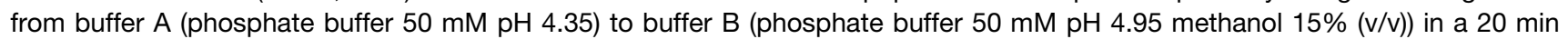
run; a modified method using organic solvents (described below in the LC-MS analysis methods) was used for analysis D-Met muropeptides for a better separation of the peaks and to avoid co-elution of muropeptides. Identity of the peaks was assigned by comparison of the retention times and profiles to other chromatograms in which mass spectrometry data had been collected, and by mass spectrometry when necessary. The relative amount of each muropeptide was calculated by dividing the peak-area of a muropeptide by the total area of the chromatogram. The apparent density of PG (PG content per cell) was assessed by normalizing the total area of the chromatogram to the $\mathrm{OD}_{600}$ of the culture used for the $\mathrm{PG}$ purification. The degree of cross-linking was calculated as described previously (Glauner et al., 1988) and as a rough estimation of D,D-cross-linkage the ratio between the tetra-tetra dimer (D44) and the tetra monomer (M4) was calculated. All PG analyses were performed using biological triplicates.

\section{Protein overexpression and purification}

vc2153 and the genes encoding for the cytoplasmic L,D-carboxypeptidases of E. coli (LdcA), A. hydrophila (AHA_1477) P. mirabilis (PMI1557) and S. enterica (STM1800) were cloned into pET28b and vca0337 into pET22b (Novagen) for expression in E.coli BL21(DE3) cells (primers used for cloning are listed in Table S4). Expression was induced in LB cultures at exponential phase with $1 \mathrm{mM}$ isopropyl $\beta$-D-thiogalactopyranoside (IPTG) for $2 \mathrm{~h}$. Cells were harvested, resuspended in $150 \mathrm{mM}$ Tris $\mathrm{HCl} \mathrm{pH} 7.5$, $150 \mathrm{mM} \mathrm{NaCl}$, and stored at $-20^{\circ} \mathrm{C}$. After thawing on ice, cells were disrupted by passaging through a French press twice. $6 \times$ His-tagged proteins were purified from cleared lysates (30 min, 50,000 rpm) on nickel-nitrilotriacetic acid-agarose columns (QIAGEN), and eluted with $150 \mathrm{mM}$ Tris $\mathrm{HCl} \mathrm{pH} \mathrm{7.5,} 150 \mathrm{mM} \mathrm{NaCl}, 250 \mathrm{mM}$ imidazole. The eluate was dialyzed for $12 \mathrm{~h}$ in $50 \mathrm{mM}$ Tris- $\mathrm{HCl} \mathrm{pH} \mathrm{7.5,} 100 \mathrm{mM} \mathrm{NaCl}$. Purified proteins were visualized by SDS-PAGE and Coomassie Brilliant Blue staining and quantified by Bio-Rad Protein Assay (Bio-Rad).

\section{In vitro protein reactions}

For pure muropeptide substrates isolation, V. cholerae or E. coli peptidoglycan was digested with muramidase (see method above) and used for purification of muropeptides by collection of HPLC-separated muropeptide peaks. For isolation of anhydro- or D-Metmodified muropeptides, in vitro reactions were performed using purified Slt70 and LdtA proteins respectively (Cava et al., 2011; Lee et al., 2013). Acetonitrile and formic acid were used as organic solvents for peak separation. Collected peaks were lyophilized, dissolved in water, and stored at $-20^{\circ} \mathrm{C}$.

In vitro reactions were prepared in a final volume of $50 \mu \mathrm{L}$ containing as substrates either pure muropeptides (10 $\mu \mathrm{g})$, intact sacculi or muramidase-digested sacculi; $10 \mu \mathrm{g}$ of purified protein ( $50 \mathrm{mM}$ final concentration) in Tris-HCl pH 8 buffer was used. To test the activity of LdcA and LdcV on high molecular weight PG, SDS-free sacculi were incubated with purified proteins and after inactivation by heating at $100^{\circ} \mathrm{C}$ for $15 \mathrm{~min}$, samples were centrifuged, and the pellet was digested with muramidase. Reactions were performed for $60 \mathrm{~min}$ at $37^{\circ} \mathrm{C}$, heat inactivated $\left(100^{\circ} \mathrm{C}, 15 \mathrm{~min}\right)$ and centrifuged $(15,000 \mathrm{rpm}, 10 \mathrm{~min})$ to remove precipitated material. Peptides in the supernatant were monitored by UPLC, identified using standard controls based on their retention time, and confirmed by UPLCMS analysis.

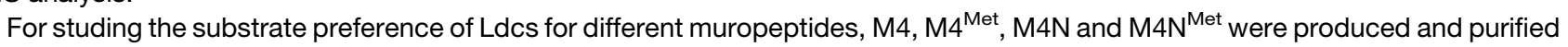
as described above. Then a mixture of the four muropeptides (containing approximately equal amounts of each of them) was used as substrate in a reaction of $150 \mu \mathrm{L}$ final volume in $50 \mathrm{mM}$ Tris- $\mathrm{HCl} \mathrm{pH} 8$ buffer and $50 \mu \mathrm{g}$ of purified Ldc protein. After addition of the protein, samples were incubated at $37^{\circ} \mathrm{C}$ and small aliquotes were removed at several incubation times, boiled to inactivate the protein and centrifuged. Soluble products were injected into the UPLC. The ratio of each muropeptide at each time-point was calculated by dividing its area by the total area calculated for all the muropeptides (substrates and products).

\section{Whole genome sequencing}

Genomic DNA was extracted using the GeneJET Genomic DNA Purification Kit and quantified with Qubit dsDNA HS Assay Kit (Thermo Scientific). $1 \mathrm{ng}$ of each DNA was used to generate the genomic libraries following the manufacturer's recommendations (Nextera XT DNA Sample Preparation Kit, Illumina). DNA libraries were then pooled in equimolar proportions and sequenced employing a MiSeq Reagent Kit V2 (Illumina). Paired-end 2x300 bp reads were generated on an Illumina MiSeq instrument. Sequences of the isogenic wild-type strain N16961 and the $\Delta v c 2153$ mutant were determined in parallel.

The sequences were analyzed using the open, web-based computational platform Galaxy (https://usegalaxy.org/) (Afgan et al., 2016) as indicated in Alvarez et al. (2018). Mapping trimmed reads to the reference V. cholerae N16961 genome was performed by the BWA-MEM (Li and Durbin, 2009) algorithm. After read alignment, Picard tools were used for the parent strains and each suppressor to mark and remove duplicate sequences mapping to different regions. Subsequently, SNPs (single-nucleotide polymorphisms) and indels (insertions and deletions) were detected by comparison of the results obtained after detecting genetic variants by FreeBayes (Garrison and Marth, 2012) and VarScan (Koboldt et al., 2012). Mapping of the larger deletion in the suppressor mutant $\mathrm{Sup}_{4}$ was carried out using the Integrative Genome Viewer (IGV) (Thorvaldsdóttir et al., 2013). 


\section{Transposon insertion sequencing}

$V$. cholerae transposon insertion libraries, of $\sim 150,000$ insertion mutants each, were generated in N16961 and in the $\Delta v c 2153$ mutant as described previously (Dörr et al., 2016) using the Himar delivery vector pSC189 (Chiang and Rubin, 2002). Transposon mutants were plated directly onto $\mathrm{LB}_{10}$ (synthetic lethality) or $\mathrm{LB}_{0}$ (suppressors analysis) agar plates containing Sm and Km. Transposon insertion sequencing was performed as described previously (Chao et al., 2013) using an Illumina MiSeq benchtop sequencer. Data analysis was conducted as described previously (Chao et al., 2013; Pritchard et al., 2014). Visual inspection of transposon insertion profiles was performed with the Sanger Artemis Genome Browser and Annotation tool (Rutherford et al., 2000).

\section{Analysis of soluble muropeptide pools}

Sample preparation to determine the level of the different soluble muropeptides was performed following the protocol described previously by Lee et al. (2016) with some modifications. Briefly, bacteria were grown until exponential phase $\left(\mathrm{OD}_{600} \sim 0.7\right)$, cooled on ice for $10 \mathrm{~min}$ and then, after adjusting the $\mathrm{OD}_{600}$ of the cultures (to have the same number of bacteria in each sample), normalized volumes of cells were harvested by centrifugation at $4,000 \mathrm{rpm}, 4^{\circ} \mathrm{C}$ for $20 \mathrm{~min}$. For analysis of the extracellular soluble muropeptides (ESM) normalized volumes of supernatants were collected, boiled for $15 \mathrm{~min}$, centrifuged to remove precipitated material and stored at $-20^{\circ} \mathrm{C}$. The cell pellets were gently resuspended and washed with ice-cold $0.9 \% \mathrm{NaCl}$ solution. After pelleting the cells again by centrifugation, they were resuspended in the remaining volume and boiled in water for $15 \mathrm{~min}$. Samples were centrifuged to remove cell debris at $14,000 \mathrm{rpm}$ for $15 \mathrm{~min}$, and soluble fractions (containing intracellular soluble muropeptides, ISM) were transferred to new tubes and stored at $-20^{\circ} \mathrm{C}$. Both, ESM and ISM samples were filtered with $0.2 \mu \mathrm{m}$ pore size filters, dried by speed vacuum, resuspended in water and used for LC-MS analyses. Soluble muropeptide analyses were performed on biological triplicates.

Detection and characterization of soluble muropeptides by LC-MS was performed on an UPLC system interfaced with a Xevo G2/XS Q-TOF mass spectrometer (Waters Corp.). Chromatographic separation was achieved as described previously (Alvarez et al., 2016) using an ACQUITY UPLC BEH C18 Column (Waters Corp.) heated at $45^{\circ} \mathrm{C}$. $0.1 \%$ formic acid in Mili-Q water (Buffer A) $0.1 \%$ formic acid in acetonitrile (buffer B) were used as eluents. The gradient of buffer B was set as follows: $0-3$ min $5 \%, 3-6$ min $5 \%-6.8 \%, 6-$ $7.5 \mathrm{~min} 6.8 \%-9 \%, 7.5-9 \mathrm{~min} 9 \%-14 \%, 9-11 \mathrm{~min} 14 \%-20 \%, 11-12 \mathrm{~min}$ hold at $20 \%$ with a flow rate of $0.175 \mathrm{~mL} \mathrm{~min}^{-1} ; 12-12.1 \mathrm{~min}$ $20 \%-90 \%, 12.1-13.5 \mathrm{~min}$ hold at $90 \%, 13.5-13.6 \mathrm{~min} 90 \%-2 \%, 13.6-16 \mathrm{~min}$ hold at $2 \%$ with a flow rate of $0.3 \mathrm{~mL} \mathrm{~min}^{-1}$; and then $16-18 \mathrm{~min}$ hold at $2 \%$ with a flow rate of $0.25 \mathrm{~mL} \mathrm{~min}^{-1}$. Chromatograms were recorded at $204 \mathrm{~nm}$. The QTOF-MS instrument was operated in positive ionization mode. Detection of ISM and ESM was in general performed by $\mathrm{MS}^{\mathrm{e}}$ to allow the acquisition of precursor and product ion data simultaneously without pre-selection of targeted molecules. For $\mathrm{MS}^{\mathrm{e}}$ the following parameters were set for ESI: capillary voltage at $3.0 \mathrm{kV}$, source temperature to $120^{\circ} \mathrm{C}$, desolvation temperature to $350^{\circ} \mathrm{C}$, sample cone voltage to $40 \mathrm{~V}$, cone gas flow $100 \mathrm{~L} \mathrm{~h}^{-1}$ and desolvation gas flow $500 \mathrm{~L} \mathrm{~h}^{-1}$. Detection of D-Met modified tetrapeptides was achieved by targeted MS, by setting the collision energy to scan between $6 \mathrm{eV}$ and 15-40 eV. Mass spectra were acquired at a speed of $0.25 \mathrm{~s} / \mathrm{scan}$. The scan was in a range of 100-2000 m/z. Data acquisition and processing was performed using UNIFI software package (Waters Corp.).

The molecular structure of each anhydro-muropeptide and the PG precursor molecules was obtained using ChemSketch (https:// www.acdlabs.com) to build a compound library in UNIFI. This compound library was used for processing the data, to detect and identify each molecule. Subsequent identification and confirmation of each muropeptide was performed by comparison of the retention-times and mass spectrometric data of experimental samples to purified authentic standards when available. Quantification was done by integrating peak areas from extracted ion chromatograms (EICs) of the corresponding $\mathrm{m} / \mathrm{z}$ value of each muropeptide.

\section{Lipid II extraction and LC-MS analysis}

Lipid extraction was performed according to the protocol described by Qiao et al. (2017) for "large-scale lipid extraction" but using only $500 \mathrm{~mL}$ cultures. Strains were grown in $\mathrm{LB}$ at $37^{\circ} \mathrm{C}$ until an $\mathrm{OD}_{600}$ of $0.4-0.6$ or 2 for exponential or stationary phase samples respectively. After the chloroform/methanol extraction steps, dried recovered interface fractions were resuspended in DMSO. The lipid tail of the extracted Lipid II was removed by ammonium acetate treatment before LC-MS analysis that was performed by targeted MS using the same parameters indicated above for analyzing soluble-muropeptides pools.

M4 ${ }^{\text {Met }}$ incorporation into the PG by recycling

$\mathrm{M} 4 \mathrm{~N}^{\mathrm{Met}}$ muropeptides were generated by initially obtaining M4N from SDS-free $V$. cholerae sacculi digested with purified $E$. coli His6tagged Slt70 protein (Höltje et al., 1975; Lee et al., 2013) and then replacing the terminal D-Ala by D-Met via in vitro L,D-transpeptidation mediated by purified LdtA protein in the presence of $20 \mathrm{mM}$ D-Met (Cava et al., 2011). Around $15 \mu \mathrm{g}$ of M4N ${ }^{\mathrm{Met}}$ (in water) or the same volume of water as negative control were added to $200 \mu \mathrm{L}$ of concentrated exponential cultures (containing $\sim 10^{9}$ bacteria) of $\Delta / d t A \Delta / d t B \Delta b s r V \Delta / d c V(\Delta 4)$ and $\Delta / d t A \Delta / d t B \Delta b s r V \Delta a m p G \Delta / d c V(\Delta 5)$ strains grown in M9 minimal medium with glucose as a carbon source. After incubation of the samples for $45 \mathrm{~min}$ at $37^{\circ} \mathrm{C}$, bacteria were pelleted and boiled in $2.5 \%$ of SDS for sacculi isolation and muramidase digestion. Solubilized muropeptides were reduced and after adjusting the $\mathrm{pH}$, injected into the UPLC/MS for the search of $\mathrm{M} 4{ }^{\mathrm{Met}}$ muropeptides by targeted MS.

\section{Competition assays}

In vitro competition indices $(\mathrm{Cl})$ were determined from cultures containing the $\Delta / d c V$ strain $\left(\right.$ lac $\left.Z^{+}\right)$carrying the empty pBAD ${ }_{18}$ vector and the $\Delta / d c V$ (lacZ) with the specified $\mathrm{pBAD}_{18}:$ :Mur-construction, mixed in a ratio 5:1. The competition assays were performed by 


\section{CellPress

incubating the cultures in $\mathrm{LB}_{0}$ (and appropriate antibiotics) supplemented with $0.2 \%$ of arabinose for $4 \mathrm{~h}$ at $37^{\circ} \mathrm{C}$. After this time, $\mathrm{CFUs}$ were counted by plating serial log dilutions on $\mathrm{LB}_{0}$ agar containing $\mathrm{Sm}, \mathrm{Km}, 0.2 \%$ arabinose and X-gal $40 \mu \mathrm{g} \mathrm{ml}{ }^{-1}$. The Cl was defined as the number of white colonies (strains overexpressing Mur proteins)/number of blue colonies (empty vector) counted after the incubation, divided by the white/blue ratio measure in the inoculum. The competition assays were performed in triplicates. In order to ensure protein overexpression from the pBAD promoter, total protein extracts obtained from arabinose induced cultures were prepared and the $6 \times$ His-tagged produced proteins were monitored by western blotting using standard techniques and an anti-His antibody.

\section{QUANTIFICATION AND STATISTICAL ANALYSIS}

GraphPad Prism 6 software was used for graphing data and statistical analysis. Details of statistical tests and the number of replicates used for each analysis can be found in the figure legends. 